\title{
SMALL-ENERGY ANALYSIS FOR THE SELFADJOINT MATRIX SCHRÖDINGER OPERATOR ON THE HALF LINE. II
}

\author{
Tuncay Aktosun \\ Department of Mathematics \\ University of Texas at Arlington \\ Arlington, TX 76019-0408, USA \\ aktosun@uta.edu \\ Martin Klaus \\ Department of Mathematics \\ Virginia Tech \\ Blacksburg, VA 24061, USA \\ mklaus@math.vt.edu \\ Ricardo Weder ${ }^{\dagger}$ \\ Departamento de Física Matemática \\ Instituto de Investigaciones en Matemáticas Aplicadas y en Sistemas \\ Universidad Nacional Autónoma de México \\ Apartado Postal 20-726, IIMAS-UNAM, México DF 01000, México \\ weder@unam.mx
}

\begin{abstract}
The matrix Schrödinger equation with a selfadjoint matrix potential is considered on the half line with the most general selfadjoint boundary condition at the origin. When the matrix potential is integrable and has a second moment, it is shown that the corresponding scattering matrix is differentiable at zero energy. An explicit formula is provided for the derivative of the scattering matrix at zero energy. The previously established results when the potential has only the first moment are improved when the second moment exists, by presenting the small-energy asymptotics for the related Jost matrix, its inverse, and various other quantities relevant to the corresponding direct and inverse scattering problems.
\end{abstract}

Mathematics Subject Classification (2010): 34L25 34L40 81U05 81Uxx

Keywords: matrix Schrödinger equation, selfadjoint boundary condition, Jost matrix, small-energy limit, scattering matrix, Jost solution, quantum wires, quantum graphs Short title: Half-line matrix Schrödinger equation

$\dagger$ Fellow Sistema Nacional de Investigadores 


\section{INTRODUCTION}

Consider the matrix Schrödinger equation on the half line

$$
-\psi^{\prime \prime}+V(x) \psi=k^{2} \psi, \quad x \in \mathbf{R}^{+},
$$

where $\mathbf{R}^{+}:=(0,+\infty)$, the prime denotes the $x$-derivative, the potential $V$ is an $n \times n$ matrix-valued function of $x$, and the wavefunction $\psi$ is either a column vector with $n$ components or a matrix of size $n \times n$. Here, $n$ is any fixed positive integer. The matrix potential $V$ is assumed to be selfadjoint, i.e.

$$
V(x)=V(x)^{\dagger},
$$

where the dagger denotes the adjoint (complex conjugate and matrix transpose), and hence $V$ is not necessarily real valued when $n \geq 2$. We further assume that $V$ belongs to class

$L_{2}^{1}\left(\mathbf{R}^{+}\right)$. By $V \in L_{j}^{1}\left(\mathbf{R}^{+}\right)$we mean that each entry of the matrix $V$ is Lebesgue measurable on $\mathbf{R}^{+}$and

$$
\int_{0}^{\infty} d x(1+|x|)^{j}|| V(x) \|<+\infty,
$$

where $\|V(x)\|$ denotes a matrix norm. Since all matrix norms are equivalent, without loss of generality, we can use the matrix norm defined as

$$
\|V(x)\|:=\max _{l} \sum_{s=1}^{n}\left|V_{l s}(x)\right|, \quad l=1, \ldots, n,
$$

with $V_{l s}(x)$ denoting the $(l, s)$-entry of the matrix $V(x)$.

We use $\mathbf{R}$ for the real axis, $\mathbf{C}$ for the complex plane, $\mathbf{C}^{+}$for the open upper-half complex plane, $\overline{\mathbf{C}^{+}}$for $\mathbf{C}^{+} \cup \mathbf{R}$, an overdot for the $k$-derivative, and $I_{n}$ for the $n \times n$ identity matrix. By a generic constant $c$, we mean a constant that does not necessarily take the same value in each appearance.

We are interested in studying (1.1) under the most general selfadjoint boundary condition at $x=0[5,16-18,21,22]$, which can be stated as

$$
-B^{\dagger} \psi(0)+A^{\dagger} \psi^{\prime}(0)=0,
$$


where the constant $n \times n$ matrices $A$ and $B$ satisfy

$$
B^{\dagger} A=A^{\dagger} B, \quad A^{\dagger} A+B^{\dagger} B>0
$$

i.e. $A^{\dagger} B$ is selfadjoint and the selfadjoint matrix $\left(A^{\dagger} A+B^{\dagger} B\right)$ is positive.

By considering the Schrödinger operator corresponding to (1.1) with a selfadjoint potential in $L_{2}^{1}\left(\mathbf{R}^{+}\right)$and with the selfadjoint boundary condition (1.3), our primary goal is to establish the small- $k$ asymptotics of various quantities related to (1.1) such as certain scattering solutions, the Jost matrix, the inverse of the Jost matrix, and the scattering matrix. In [5], which is considered to be part I, we have provided the corresponding small$k$ analysis under the assumption that $V$ is selfadjoint and belongs to $L_{1}^{1}\left(\mathbf{R}^{+}\right)$. In the current paper, which is considered to be part II and a sequel to [5], we refine those asymptotics by using the stronger assumption $V \in L_{2}^{1}\left(\mathbf{R}^{+}\right)$instead of $V \in L_{1}^{1}\left(\mathbf{R}^{+}\right)$. Such an analysis is crucial in the study of the corresponding direct and inverse scattering problems. Without knowing the behavior of the scattering data at and near $k=0$, it is not possible to solve the relevant inverse scattering problem. For the corresponding large- $k$ asymptotics of the Jost matrix and of the scattering matrix, we refer the reader to [6] and the references therein.

The direct scattering problem for (1.1) consists of the determination of the scattering matrix and the bound-state information when the matrix potential $V$ and the selfadjoint boundary condition (1.3) are known. On the other hand, the corresponding inverse scattering problem is to recover the potential and the boundary condition from an appropriate set of scattering data. We recall that a bound state for the corresponding Schrödinger operator is an $n \times 1$ matrix-valued solution to (1.1), satisfying the boundary condition (1.3), and having each entry square integrable in $x \in \mathbf{R}^{+}$.

The small- $k$ analysis of the scattering matrix is not easy even in the scalar case. For example, in the full-line scalar case Deift and Trubowitz [10] stated that the characterization of the scattering data given by Faddeev [12] might not hold and in fact even the 
continuity of the scattering matrix was not clear when the real-valued potential belonged to $L_{1}^{1}(\mathbf{R})$, and they introduced the stronger assumption that the potential belonged to $L_{2}^{1}(\mathbf{R})$. The proof of the continuity of the scattering matrix was given later in the scalar case when $L_{1}^{1}(\mathbf{R})$; for details we refer the reader to $[19,20]$ and the references therein. The techniques needed for the small- $k$ analysis especially in the exceptional case, i.e. when the Jost matrix is not invertible, are very delicate and hence a careful analysis is needed.

Besides its mathematical significance, our analysis is also important due to its direct relevance to applications in quantum mechanical scattering of particles with internal structures, scattering on graphs [7,8,11,13-15,23-26], and quantum wires [21,22]. For example, the problem under study describes $n$ connected very thin quantum wires forming a onevertex graph with open ends. A linear boundary condition is imposed at the vertex and the behavior on each wire is governed by a Schrödinger operator. The corresponding direct and inverse problems have physical relevance in designing elementary gates in quantum computing and nanotubes for microscopic electronic devices, with strings of atoms forming a star-shaped graph. For further details regarding applications we refer the reader to [21] and the references therein.

Our paper complements the study by Agranovich and Marchenko [1], where the inverse scattering problem is analyzed with attention to the behavior at $k=0$, but only under the Dirichlet boundary condition, i.e. when $A=0$ in (1.3) so that the boundary condition becomes $\psi(0)=0$. It also complements the study by Harmer [16-18] where the general selfadjoint boundary condition equivalent to (1.3) is used to investigate the inverse problem for (1.1) but without any corresponding small- $k$ analysis. We refer the reader to [2,20] for the small- $k$ analysis for the half-line scalar Schrödinger equation, to [3,19] for the full-line scalar Schrödinger equation, to [4] for the full-line matrix Schrödinger equation, and to [5] for the half-line matrix Schrödinger equation with the most general selfadjoint boundary condition at the origin under the weaker assumption $V \in L_{1}^{1}\left(\mathbf{R}^{+}\right)$. 
Our paper is organized as follows. In Section 2 we introduce three $n \times n$ matrixvalued solutions to (1.1) and provide their relevant properties that are needed later on. In Section 3 we obtain the small- $k$ asymptotics of the key quantity $P(k)$ defined in (3.1), and in Theorem 3.1 we provide the explicit asymptotics as $k \rightarrow 0$ in $\overline{\mathbf{C}^{+}}$for $P(k)$ up to $o\left(k^{2}\right)$. In Section 3 we also present a remarkable result for the small- $k$ asymptotics of $f^{\prime}(k, x) f(k, x)^{-1}$ at any fixed $x \in \mathbf{R}^{+}$for which $f(0, x)^{-1}$ exists, as well as a similar result for the small- $k$ asymptotics of $f(k, x) f^{\prime}(k, x)^{-1}$ at any fixed $x \in \mathbf{R}^{+}$for which $f^{\prime}(0, x)^{-1}$ exists. The corresponding asymptotic expansions are remarkable in the sense that they are obtained explicitly up to $o\left(k^{2}\right)$ even though the small- $k$ asymptotics can only be provided up to $o(k)$ for the individual matrices $f(k, x)$ and $f^{\prime}(k, x)$ and their inverses. In Section 4 we introduce the Jost matrix $J(k)$ and the scattering matrix $S(k)$. In Section 5 we analyze the small- $k$ asymptotics of $J(k), J(k)^{-1}$, and $S(k)$ in the generic case, i.e. when $J(0)^{-1}$ exists; we prove the differentiability at $k=0$ for those three matrices, and we provide their explicit expansions up to $o(k)$ as $k \rightarrow 0$. In Section 6 we present a $2 n \times 2 n$ matrix that is always invertible and that is closely related to $J(0)$; the result is remarkable in the sense that the invertibility holds even when the $n \times n$ matrix $J(0)$ is not invertible. In Section 7 we analyze the small- $k$ asymptotics in the exceptional case, i.e. when $J(0)^{-1}$ does not exist, and we show that, when the potential $V$ in (1.1) is selfadjoint and belongs to $L_{2}^{1}\left(\mathbf{R}^{+}\right)$, the corresponding matrices $J(k), k J(k)^{-1}$, and $S(k)$ are differentiable at $k=0$; we also provide the explicit asymptotics up to $o(k)$ for those three matrices as $k \rightarrow 0$. Finally, in Section 8 we illustrate our results on the small- $k$ asymptotics of the Jost matrix, the inverse of the Jost matrix, and the scattering matrix by presenting an explicit example.

\section{PROPERTIES OF SOLUTIONS}

In this section we introduce three $n \times n$ matrix-valued solutions to (1.1) denoted by $f(k, x), \omega(k, x)$, and $\varphi(k, x)$, respectively, and we present their relevant properties that are needed later on. 
By taking the matrix adjoint of (1.1) and using the selfadjointness (1.2) of the potential $V$, we obtain the adjoint Schrödinger equation

$$
-\phi^{\prime \prime}+\phi V(x)=k^{2} \phi, \quad x \in \mathbf{R}^{+}
$$

If $\psi(k, x)$ is a solution to (1.1) for a real value of the parameter $k$, then $\psi(k, x)^{\dagger}$ and $\psi(-k, x)^{\dagger}$ are solutions to $(2.1)$. Furthermore, if $\psi(k, x)$ has an analytic extension from $k \in \mathbf{R}$ to $k \in \mathbf{C}^{+}$, then $\psi\left(-k^{*}, x\right)^{\dagger}$ also has an analytic extension from $k \in \mathbf{R}$ to $k \in \mathbf{C}^{+}$, where we us an asterisk to denote complex conjugation.

Let $[F ; G]:=F G^{\prime}-F^{\prime} G$ denote the Wronskian. It is known $[4,5]$ that for any two $n \times n$ matrix-valued solutions $\psi(k, x)$ and $\Psi(k, x)$ to $(1.1)$, the Wronskians $\left[\Psi(k, x)^{\dagger} ; \psi(k, x)\right]$ for real $k$-values is independent of $x$. In case $\psi(k, x)$ and $\Psi(k, x)$ have analytic extensions from $k \in \mathbf{R}$ to $k \in \mathbf{C}^{+}$, the Wronskian $\left[\Psi\left(-k^{*}, x\right)^{\dagger} ; \psi(k, x)\right]$ is independent of $x$.

The Jost solution to (1.1) is the $n \times n$ matrix-valued solution satisfying, for each fixed $k \in \overline{\mathbf{C}^{+}} \backslash\{0\}$, the spatial asymptotics

$$
f(k, x)=e^{i k x}\left[I_{n}+o(1)\right], \quad f^{\prime}(k, x)=i k e^{i k x}\left[I_{n}+o(1)\right], \quad x \rightarrow+\infty .
$$

From (1.1) and (2.2) it follows that

$$
f(k, x)=e^{i k x} I_{n}+\frac{1}{k} \int_{x}^{\infty} d y[\sin k(y-x)] V(y) f(k, y) .
$$

By letting $k \rightarrow 0$ in (2.3) we obtain

$$
f(0, x)=I_{n}+\int_{x}^{\infty} d y(y-x) V(y) f(0, y) .
$$

It can be verified that $f(0, x)$ satisfies the $n \times n$ matrix-valued zero-energy Schrödinger equation

$$
-\psi^{\prime \prime}+V(x) \psi=0, \quad x \in \mathbf{R}^{+}
$$

which is obtained from (1.1) by setting $k=0$ there, as well as the asymptotic conditions

$$
f(0, x)=I_{n}+o(1), \quad f^{\prime}(0, x)=o(1), \quad x \rightarrow+\infty
$$


which are obtained from (1.1) and (2.2), respectively, by letting $k \rightarrow 0$.

Some relevant properties associated with $f(k, x)$ and $f(0, x)$ are stated in the following two propositions.

Proposition 2.1 Assume that the potential $V$ is selfadjoint and belongs to $L_{2}^{1}\left(\mathbf{R}^{+}\right)$, and let $f(k, x)$ be the corresponding Jost solution to (1.1) appearing in (2.2). Then:

(a) For each fixed $x \in[0,+\infty)$, the quantities $f(k, x)$ and $f^{\prime}(k, x)$ are analytic in $k \in \mathbf{C}^{+}$, continuous in $k \in \overline{\mathbf{C}^{+}}$, and we have

$$
\begin{gathered}
f(k, x)=f(0, x)+k \dot{f}(0, x)+o(k), \quad k \rightarrow 0 \text { in } \overline{\mathbf{C}^{+}}, \\
f^{\prime}(k, x)=f^{\prime}(0, x)+k \dot{f}^{\prime}(0, x)+o(k), \quad k \rightarrow 0 \text { in } \overline{\mathbf{C}^{+}}
\end{gathered}
$$

where we recall that an overdot denotes the $k$-derivative.

(b) For each fixed $k \in \overline{\mathbf{C}^{+}}$, the matrix-valued functions $f(k, x), f^{\prime}(k, x), \dot{f}(k, x)$, and $\dot{f}^{\prime}(k, x)$ are continuous in $x \in[0,+\infty)$.

(c) For each fixed $k \in \overline{\mathbf{C}^{+}} \backslash\{0\}$, the spatial asymptotics in (2.2) can actually be replaced by the sharper estimates

$$
f(k, x)=e^{i k x}\left[I_{n}+o\left(\frac{1}{x^{2}}\right)\right], \quad f^{\prime}(k, x)=i k e^{i k x}\left[I_{n}+o\left(\frac{1}{x^{2}}\right)\right], \quad x \rightarrow+\infty
$$

as a result of $V \in L_{2}^{1}\left(\mathbf{R}^{+}\right)$.

(d) The Jost solution $f(k, x)$ satisfies the Wronskian relations

$$
\begin{gathered}
{\left[f( \pm k, x)^{\dagger} ; f( \pm k, x)\right]= \pm 2 i k I_{n}, \quad k \in \mathbf{R},} \\
{\left[f\left(-k^{*}, x\right)^{\dagger} ; f(k, x)\right]=0, \quad k \in \overline{\mathbf{C}^{+}} .}
\end{gathered}
$$

(e) When $x$ is large, the matrix $f(0, x)$ is invertible. 
(f) For every $a \in[0,+\infty)$ for which the matrix $f(0, a)$ is invertible, the matrix $f(k, a)^{-1}$ exists when $k$ is in a neighborhood of $k=0$ in $\overline{\mathbf{C}^{+}}$and we have

$$
f(k, a)^{-1}=f(0, a)^{-1}-k f(0, a)^{-1} \dot{f}(0, a) f(0, a)^{-1}+o(k), \quad k \rightarrow 0 \text { in } \overline{\mathbf{C}^{+}} .
$$

For every $a \in[0,+\infty)$ for which $f^{\prime}(0, a)$ is invertible, the matrix $f^{\prime}(k, a)^{-1}$ exists when $k$ is in a neighborhood of $k=0$ in $\overline{\mathbf{C}^{+}}$, and as $k \rightarrow 0$ in $\overline{\mathbf{C}^{+}}$we have

$$
f^{\prime}(k, a)^{-1}=f^{\prime}(0, a)^{-1}-k f^{\prime}(0, a)^{-1} \dot{f}^{\prime}(0, a) f^{\prime}(0, a)^{-1}+o(k) .
$$

PROOF: The proof of (a) follows from Theorem 2.1 of [4]. The proof of (b) can be given by using iteration on (2.3) and on the related integral equations obtained by taking the derivatives with respect to $x$ and $k$. Then, by representing the relevant quantities as uniformly convergent series of continuous functions, the continuity in $x$ can be established for those relevant quantities. The statement of (c) can be obtained from Theorem 1.4.1 of [1]. We quote (2.10) and (2.11) given in (d) from (3.18) and (3.19), respectively, of [5]. The invertibility in (e) is a consequence of (b) and (2.6). The existence of $f(0, a)^{-1}$, the continuity of $f(k, a)$ at $k=0$ in $\overline{\mathbf{C}^{+}}$, and (2.7) yield (2.12), and we establish (2.13) in a similar way.

The $n \times n$ matrix-valued zero-energy Schrödinger equation given in (2.5) has [4] two linearly independent $n \times n$ matrix solutions, one of which is bounded and the other is unbounded. As indicated in the following proposition, a bounded solution to (2.5) is given by $f(0, x)$ and an unbounded solution is given [4] by $\dot{f}(0, x)$. Furthermore, the $2 n$ columns of $f(0, x)$ and $\dot{f}(0, x)$ form $2 n$ linearly independent $n \times 1$ matrix-valued solutions to $(2.5)$.

Proposition 2.2 Assume that the potential $V$ is selfadjoint and belongs to $L_{2}^{1}\left(\mathbf{R}^{+}\right)$, and let $f(k, x)$ be the corresponding Jost solution to (1.1) appearing in (2.2). Then:

(a) The zero-energy Jost solution $f(0, x)$ appearing in (2.6) is a bounded solution to (2.5), and in fact it satisfies the sharper spatial asymptotics

$$
f(0, x)=I_{n}+o\left(\frac{1}{x}\right), \quad f^{\prime}(0, x)=o\left(\frac{1}{x^{2}}\right), \quad x \rightarrow+\infty .
$$


(b) We have $f(0, x)-I_{n} \in L^{1}\left(\mathbf{R}^{+}\right)$under the weaker assumption $V \in L_{1}^{1}\left(\mathbf{R}^{+}\right)$.

(c) For each fixed $x \in \mathbf{R}^{+}$, we have

$$
\begin{gathered}
\int_{x}^{\infty} d y V(y) f(0, y)=-f^{\prime}(0, x) \\
\int_{x}^{\infty} d y y V(y) f(0, y)=-I_{n}+f(0, x)-x f^{\prime}(0, x) \\
\int_{x}^{\infty} d y y^{2} V(y) f(0, y)=-x^{2} f^{\prime}(0, x)+2 x\left[f(0, x)-I_{n}\right]+2 \int_{x}^{\infty} d y\left[f(0, y)-I_{n}\right] .
\end{gathered}
$$

(d) The quantity $\dot{f}(0, x)$ is an unbounded solution to (2.5) and it satisfies the spatial asymptotics

$$
\dot{f}(0, x)=i x\left[I_{n}+o\left(\frac{1}{x}\right)\right], \quad \dot{f}^{\prime}(0, x)=i I_{n}+o\left(\frac{1}{x}\right), \quad x \rightarrow+\infty .
$$

(e) For each $x \in \mathbf{R}^{+}$, we have

$$
\left[\begin{array}{ll}
f(0, x) & \dot{f}(0, x) \\
f^{\prime}(0, x) & \dot{f}^{\prime}(0, x)
\end{array}\right]^{-1}=i\left[\begin{array}{ll}
\dot{f}^{\prime}(0, x)^{\dagger} & -\dot{f}(0, x)^{\dagger} \\
f^{\prime}(0, x)^{\dagger} & -f(0, x)^{\dagger}
\end{array}\right]
$$

PROOF: We already know (a), (b), and (d) from the Appendix of [4]. We obtain (2.19) in (e) from

$$
\left[\begin{array}{cc}
f(0, x) & \dot{f}(0, x) \\
f^{\prime}(0, x) & \dot{f}^{\prime}(0, x)
\end{array}\right]\left[\begin{array}{ll}
\dot{f}^{\prime}(0, x)^{\dagger} & -\dot{f}(0, x)^{\dagger} \\
f^{\prime}(0, x)^{\dagger} & -f(0, x)^{\dagger}
\end{array}\right]=\left[\begin{array}{cc}
-i I_{n} & 0 \\
0 & -i I_{n}
\end{array}\right],
$$

which is given in the Appendix of [4]. As for (c), we prove (2.15) and (2.16) directly by using (2.4); with the help of (2.5), (2.14), (b), and integration by parts, we get (2.17).

We define another $n \times n$ matrix-valued solution to (1.1), denoted by $\omega(k, x)$, which satisfies the initial conditions

$$
\omega(k, a)=f(0, a), \quad \omega^{\prime}(k, a)=f^{\prime}(0, a),
$$


where $a$ is the constant appearing in Proposition 2.1(f), i.e. $a$ is a nonnegative constant for which $f(0, a)^{-1}$ exists. We suppress the dependence on $a$ in our notation for $\omega(k, x)$. Note that $\omega(k, x)$ satisfies [5]

$$
\begin{aligned}
\omega(k, x)= & f(0, a) \cos k(x-a)+f^{\prime}(0, a) \frac{\sin k(x-a)}{k} \\
& +\frac{1}{k} \int_{a}^{x} d y[\sin k(x-y)] V(y) \omega(k, y) .
\end{aligned}
$$

From (2.22), by letting $k \rightarrow 0$, we obtain

$$
\omega(0, x)=f(0, a)+f^{\prime}(0, a)(x-a)+\int_{a}^{x} d y(x-y) V(y) \omega(0, y)
$$

Similar to (A19) of [4], we see that $\omega(k, x)$ satisfies

$$
\omega(k, x)=\omega(0, x)+i k^{2} f(0, x) \int_{a}^{x} d y \dot{f}(0, y)^{\dagger} \omega(k, y)+i k^{2} \dot{f}(0, x) \int_{a}^{x} d y f(0, y)^{\dagger} \omega(k, y) .
$$

Note that, with the help of (2.20) and (2.23), one can directly verify that the right-hand side of (2.24) satisfies (1.1) and (2.21).

Let us write $(2.24)$ as

$$
\omega(k, x)=\omega(0, x)+i k^{2} \omega_{1}(x)+i k^{2} \omega_{2}(k, x),
$$

where we have defined

$$
\begin{aligned}
& \omega_{1}(x):=f(0, x) \int_{a}^{x} d y \dot{f}(0, y)^{\dagger} \omega(0, y)+\dot{f}(0, x) \int_{a}^{x} d y f(0, y)^{\dagger} \omega(0, y) \\
& \omega_{2}(k, x):= f(0, x) \int_{a}^{x} d y \dot{f}(0, y)^{\dagger}[\omega(k, y)-\omega(0, y)] \\
&+\dot{f}(0, x) \int_{a}^{x} d y f(0, y)^{\dagger}[\omega(k, y)-\omega(0, y)] .
\end{aligned}
$$

Some relevant properties of $\omega(k, x)$ are provided in the following proposition.

Proposition 2.3 Assume that the potential $V$ appearing in (1.1) is selfadjoint, and let $\omega(k, x)$ be the solution to (1.1) appearing in (2.21). Then: 
(a) When $V$ belongs to $L_{1}^{1}\left(\mathbf{R}^{+}\right)$, for each fixed $x \in \mathbf{R}^{+}$the quantities $\omega(k, x)$ and $\omega^{\prime}(k, x)$ are entire in $k$, and we have

$$
\begin{gathered}
\omega(0, x)=f(0, x), \quad x \in \mathbf{R}^{+}, \\
\|\omega(k, x)-\omega(0, x)\| \leq c\left(\frac{|k|(x-a)}{1+|k|(x-a)}\right)^{2} e^{(\operatorname{Im}[k])(x-a)}, \quad k \in \overline{\mathbf{C}^{+}}, \quad x \geq a,
\end{gathered}
$$

where $c$ is a generic constant.

(b) When $V$ belongs to $L_{2}^{1}\left(\mathbf{R}^{+}\right)$, the quantity $\omega_{2}(k, x)$ defined in (2.27) satisfies

$$
\left\|\omega_{2}(k, x)\right\| \leq c\left(1+x^{2}\right)\left(\frac{|k| x}{1+|k| x}\right)^{2} e^{(\operatorname{Im}[k])(x-a)}, \quad k \in \overline{\mathbf{C}^{+}}, \quad x \geq a .
$$

(c) When $V$ belongs to $L_{2}^{1}\left(\mathbf{R}^{+}\right)$, the quantity $\omega_{2}(k, x)$ defined in (2.27) satisfies

$$
\int_{a}^{\infty} d x e^{i k x} V(x) \omega_{2}(k, x)=o(1), \quad k \rightarrow 0 \text { in } \overline{\mathbf{C}^{+}}
$$

(d) When $V$ belongs to $L_{2}^{1}\left(\mathbf{R}^{+}\right)$, the quantity $\omega_{1}(x)$ defined in (2.26) satisfies

$$
\begin{gathered}
\left\|\omega_{1}(x)\right\| \leq c\left(1+x^{2}\right), \quad x \in \mathbf{R}^{+}, \\
\int_{a}^{\infty} d x\left[e^{i k x}-1\right] V(x) \omega_{1}(x)=o(1), \quad k \rightarrow 0 \text { in } \overline{\mathbf{C}^{+}} \\
\int_{a}^{\infty} d x V(x) \omega_{1}(x)=i \int_{a}^{\infty} d x\left[f(0, x)^{\dagger} f(0, x)-I_{n}\right]-i \int_{a}^{\infty} d x\left[f(0, x)-I_{n}\right] .
\end{gathered}
$$

PROOF: The analyticity properties stated in (a) follow from the standard theory of initialvalue problems for ordinary differential equations [9]. Note that (2.28) follows from (2.21), the fact that $\omega(k, x)$ is a solution to (1.1), and the uniqueness of a corresponding initialvalue problem. The estimate in (2.29) is obtained in Proposition 5.1 of [5]. Let us now prove (2.30). From Proposition 2.1(b) we know that $f(0, x)$ and $\dot{f}(0, x)$ are continuous in $x \in \mathbf{R}^{+}$, and hence from (2.14) and (2.18) we get

$$
\|f(0, x)\| \leq c, \quad\|\dot{f}(0, x)\| \leq c(1+x), \quad x \in \mathbf{R}^{+}
$$


Using (2.29) and (2.35) in (2.27), by exploiting the fact that $z \mapsto z^{2} /(1+z)^{2}$ is an increasing function in $z \in \mathbf{R}^{+}$and $(1+x)^{2} \leq 2\left(1+x^{2}\right)$ for $x \in \mathbf{R}^{+}$, we obtain (2.30). We get (2.31) by using (2.30) and by exploiting the fact that $V \in L_{2}^{1}\left(\mathbf{R}^{+}\right)$. We obtain (2.32) by using (2.28) and (2.35) in (2.26). Using (2.32) and the fact that

$$
\left|e^{i z}-1\right| \leq \frac{c|z|}{1+|z|}, \quad z \in \overline{\mathbf{C}^{+}},
$$

we get

$$
\left\|\left(e^{i k x}-1\right) V(x) \omega_{1}(x)\right\| \leq \frac{c|k| x}{1+|k| x}\left(1+x^{2}\right)\|V(x)\|, \quad x \in \mathbf{R}^{+}, \quad k \in \overline{\mathbf{C}^{+}} .
$$

Thus, (2.36) implies (2.33) when $V \in L_{2}^{1}\left(\mathbf{R}^{+}\right)$. Let us finally prove (2.34). First, we note that the integrals on the right-hand side in (2.34) exist as a result of Proposition 2.2(c), the boundedness of $f(0, x)$ expressed in $(2.35)$, and the decomposition

$$
f(0, x)^{\dagger} f(0, x)-I_{n}=\left[f(0, x)^{\dagger}-I_{n}\right] f(0, x)+\left[f(0, x)-I_{n}\right] .
$$

Using (2.28), let us write (2.26) as

$$
\omega_{1}(x)=f(0, x) G_{1}(x)+\dot{f}(0, x) G_{2}(x),
$$

where we have defined

$$
G_{1}(x):=\int_{a}^{x} d y \dot{f}(0, y)^{\dagger} f(0, y), \quad G_{2}(x):=\int_{a}^{x} d y f(0, y)^{\dagger} f(0, y) .
$$

With the help of (2.35) we see that

$$
G_{1}(x)=O\left(x^{2}\right), \quad G_{2}(x)=(x-a) I_{n}+\int_{a}^{\infty} d y\left[f(0, y)^{\dagger} f(0, y)-I_{n}\right]+o(1), \quad x \rightarrow+\infty .
$$

Note that (2.14), (2.18), and (2.40) yield

$$
\begin{gathered}
f^{\prime}(0, x) G_{1}(x)=o(1), \quad x \rightarrow+\infty, \\
\dot{f}^{\prime}(0, x) G_{2}(x)=i(x-a) I_{n}+i \int_{a}^{\infty} d y\left[f(0, y)^{\dagger} f(0, y)-I_{n}\right]+o(1), \quad x \rightarrow+\infty .
\end{gathered}
$$


Since $f(0, x)$ and $\dot{f}(0, x)$ are solutions to (2.5), with the help of (2.38) we obtain

$$
\int_{a}^{x} d y V(y) \omega_{1}(y)=\int_{a}^{x} d y\left[f^{\prime \prime}(0, y) G_{1}(y)+\dot{f}^{\prime \prime}(0, y) G_{2}(y)\right] .
$$

Using integration by parts in (2.43), with the help of (2.38)-(2.42) and the fact that $G_{1}(a)=$ $G_{2}(a)=0$, we get

$$
\begin{aligned}
\int_{a}^{x} d y V(y) \omega_{1}(y)= & -\int_{a}^{x} d y\left[f^{\prime}(0, y) \dot{f}(0, y)^{\dagger}+\dot{f}^{\prime}(0, y) f(0, y)^{\dagger}\right] f(0, y) \\
& +i(x-a) I_{n}+i \int_{a}^{\infty} d y\left[f(0, y)^{\dagger} f(0, y)-I_{n}\right]+o(1), \quad x \rightarrow+\infty
\end{aligned}
$$

Using in (2.44) the equality from the (2,2)-entry in (2.20), we obtain (2.34).

Let us introduce the $n \times n$ matrix-valued solution $\varphi(k, x)$ to (1.1) satisfying the initial conditions

$$
\varphi(k, 0)=A, \quad \varphi^{\prime}(k, 0)=B
$$

where $A$ and $B$ are the constant matrices appearing in (1.3). The relevant properties of $\varphi(k, x)$ are summarized in the following proposition.

Proposition 2.4 Assume that the potential $V$ is selfadjoint and belongs to $L_{2}^{1}\left(\mathbf{R}^{+}\right)$, and let $\varphi(k, x)$ be the corresponding solution to (1.1) appearing in (2.45). Then:

(a) For each fixed $x \in[0,+\infty)$, the quantities $\varphi(k, x)$ and $\varphi^{\prime}(k, x)$ are entire in $k \in \mathbf{C}$.

(b) For each fixed $x \in[0,+\infty)$, we have

$$
\varphi(k, x)=\varphi(0, x)+O\left(k^{2}\right), \quad \varphi^{\prime}(k, x)=\varphi^{\prime}(0, x)+O\left(k^{2}\right), \quad k \rightarrow 0 \text { in } \mathbf{C} .
$$

PROOF: The properties listed in (a) are already known [5] and can be established by using the integral relation given in (3.7) of [5], namely

$$
\varphi(k, x)=A \cos k x+B \frac{\sin k x}{k}+\frac{1}{k} \int_{0}^{x} d y[\sin k(x-y)] V(y) \varphi(k, y) .
$$

The proof of (b) can be given by using the integral relation

$$
\varphi(k, x)=\varphi(0, x)+i k^{2} f(0, x) \int_{0}^{x} d y \dot{f}(0, y)^{\dagger} \varphi(k, y)+i k^{2} \dot{f}(0, x) \int_{0}^{x} d y f(0, y)^{\dagger} \varphi(k, y)
$$


which, like (2.24), follows by using the method of variation of parameters.

\section{PRELIMINARIES}

With the goal of obtaining small- $k$ asymptotics of the Jost matrix, its inverse, and the scattering matrix, in this section we provide the small- $k$ analysis for certain quantities related to (1.1). Our first main result is given in Theorem 3.1 and it provides the asymptotics up to $o\left(k^{2}\right)$ as $k \rightarrow 0$ in $\overline{\mathbf{C}^{+}}$for the key $n \times n$ matrix-valued quantity $P(k)$ defined in (3.1). Our second main result is given in Theorem 3.2, which establishes the expansions up to $o\left(k^{2}\right)$ as $k \rightarrow 0$ in $\overline{\mathbf{C}^{+}}$for the matrices $f^{\prime}(k, x) f(k, x)^{-1}$ and $f(k, x) f^{\prime}(k, x)^{-1}$.

As in (5.27) of [5], in terms of a Wronskian let us define

$$
P(k):=\left[\omega\left(-k^{*}, x\right)^{\dagger} ; f(k, x)\right]
$$

where $f(k, x)$ and $\omega(k, x)$ are the solutions to (1.1) appearing in (2.2) and (2.21), respectively. Note that $P(k)$ depends on the choice of $a$ because, as apparent from $(2.21), \omega(k, x)$ depends on $a$. However, we have suppressed $a$ in our notation of $P(k)$. The Wronskian in (3.1) is independent of $x$, and its value can be found by using $x=a$ in (3.1) to obtain

$$
P(k)=f(0, a)^{\dagger} f^{\prime}(k, a)-f^{\prime}(0, a)^{\dagger} f(k, a),
$$

where we have used (2.21).

In the following theorem we state some relevant properties of $P(k)$, which are needed in determining the small- $k$ asymptotics of the Jost matrix $J(k)$ defined in (4.1).

Theorem 3.1 Assume that the potential $V$ is selfadjoint and belongs to $L_{2}^{1}\left(\mathbf{R}^{+}\right)$, and let $f(k, x)$ be the Jost solution to (1.1) satisfying (2.2). Then, the matrix $P(k)$ given in (3.1) is analytic in $\mathbf{C}^{+}$, continuous in $\overline{\mathbf{C}^{+}}$, and satisfies

$$
P(k)=i k I_{n}+k^{2}\left(-a I_{n}+\int_{a}^{\infty} d y\left[f(0, y)^{\dagger} f(0, y)-I_{n}\right]\right)+o\left(k^{2}\right), \quad k \rightarrow 0 \text { in } \overline{\mathbf{C}^{+}}
$$


PROOF: The analyticity in $\mathbf{C}^{+}$and the continuity in $\overline{\mathbf{C}^{+}}$follow from Proposition 2.1(a) and Proposition 2.3(a), namely, the fact that $f(k, x)$ and $f^{\prime}(k, x)$ satisfy those properties for each fixed $x \in \mathbf{R}^{+}$and that $\omega(k, x)$ and $\omega^{\prime}(k, x)$ are entire in $k$ for each fixed $x \in \mathbf{R}^{+}$. Let us note that the convergence of the integral in (3.3) is a result of (2.37) and Proposition 2.2(b). In order to establish (3.3), we could try to use the Maclaurin expansions in $k$ for $\omega(k, x)$ and $\omega^{\prime}(k, x)$ and the expansions in (2.3) and (2.8). However, we then only get $P(k)=i k I_{n}+o(k)$ in (3.3), which is already valid [5] under the weaker assumption $V \in L_{1}^{1}\left(\mathbf{R}^{+}\right)$. Thus, we have to use more sophisticated techniques to establish the expansion in (3.3). We proceed as follows. Differentiating (2.22) we get

$$
\begin{aligned}
\omega^{\prime}(k, x)= & -k f(0, a) \sin k(x-a)+f^{\prime}(0, a) \cos k(x-a) \\
& +\int_{a}^{x} d y[\cos k(x-y)] V(y) \omega(k, y) .
\end{aligned}
$$

Using (2.22) and (3.4) on the right-hand side of (3.1), with the help of (1.2) we obtain

$$
P(k)=P_{1}(k, x)+P_{2}(k, x)+P_{3}(k, x)+P_{4}(k, x)+P_{5}(k, x)+P_{6}(k, x),
$$

where we have defined

$$
\begin{gathered}
P_{1}(k, x):=[\cos k(x-a)] f(0, a)^{\dagger} f^{\prime}(k, x) \\
P_{2}(k, x):=\frac{\sin k(x-a)}{k} f^{\prime}(0, a)^{\dagger} f^{\prime}(k, x) \\
P_{3}(k, x):=k[\sin k(x-a)] f(0, a)^{\dagger} f(k, x) \\
P_{4}(k, x):=-[\cos k(x-a)] f^{\prime}(0, a)^{\dagger} f(k, x), \\
P_{5}(k, x):=\int_{a}^{x} d y \omega(0, y)^{\dagger} V(y)\left[\frac{\sin k(x-y)}{k} f^{\prime}(k, x)-[\cos k(x-y)] f(k, x)\right] \\
P_{6}(k, x):= \\
\int_{a}^{x} d y\left[\omega\left(-k^{*}, y\right)^{\dagger}-\omega(0, y)^{\dagger}\right] V(y)\left[\frac{\sin k(x-y)}{k} f^{\prime}(k, x)-[\cos k(x-y)] f(k, x)\right] .
\end{gathered}
$$

Since the value of $P(k)$ defined in (3.1) is independent of $x$, we will evaluate the right-hand side in (3.5) as $x \rightarrow+\infty$. With this goal in mind, we proceed as follows. Writing the sine 
and cosine functions in (3.6)-(3.9) in terms of complex exponentials $e^{i k(x-a)}$ and $e^{-i k(x-a)}$ and using (2.9), from (3.6)-(3.9) we obtain

$$
\begin{aligned}
P_{1}(k, x)+P_{2}(k, x)+ & P_{3}(k, x)+P_{4}(k, x) \\
& =\left[i k e^{i k a} f(0, a)^{\dagger}-e^{i k a} f^{\prime}(0, a)^{\dagger}\right]+o(1), \quad x \rightarrow+\infty .
\end{aligned}
$$

Proceeding in a similar manner, with the help of (2.9), as $x \rightarrow+\infty$ from (3.10) and (3.11) we obtain

$$
\begin{gathered}
P_{5}(k, x)=-\int_{a}^{\infty} d y \omega(0, y)^{\dagger} V(y) e^{i k y}+o(1), \quad x \rightarrow+\infty, \\
P_{6}(k, x)=-\int_{a}^{\infty} d y\left[\omega\left(-k^{*}, y\right)^{\dagger}-\omega(0, y)^{\dagger}\right] V(y) e^{i k y}+o(1), \quad x \rightarrow+\infty .
\end{gathered}
$$

Next we will estimate the integrals in (3.13) and (3.14) further, by working on (3.13) first. We decompose the integral in (3.13) as

$$
P_{5}(k, x)=P_{7}(k)+P_{8}(k)+o(1), \quad x \rightarrow+\infty,
$$

where we have defined

$$
\begin{gathered}
P_{7}(k):=-\int_{a}^{\infty} d y \omega(0, y)^{\dagger} V(y)\left[1+i k y-\frac{k^{2} y^{2}}{2}\right], \\
P_{8}(k):=-\int_{a}^{\infty} d y \omega(0, y)^{\dagger} V(y)\left[e^{i k y}-1-i k y+\frac{k^{2} y^{2}}{2}\right] .
\end{gathered}
$$

With the help of (2.14)-(2.17) and (2.28), we can write $P_{7}(k)$ as

$$
P_{7}(k)=p_{1}-i k p_{2}+k^{2} p_{3},
$$

where we have defined

$$
\begin{gathered}
p_{1}:=f^{\prime}(0, a)^{\dagger} \\
p_{2}:=-I_{n}+f(0, a)^{\dagger}-a f^{\prime}(0, a)^{\dagger} \\
p_{3}:=-\frac{1}{2} a^{2} f^{\prime}(0, a)^{\dagger}+a\left[f(0, a)^{\dagger}-I_{n}\right]+\int_{a}^{\infty} d y\left[f(0, y)^{\dagger}-I_{n}\right] .
\end{gathered}
$$


Note that for any $z \in \overline{\mathbf{C}^{+}}$we have

$$
\left|e^{i z}-1-i z+\frac{z^{2}}{2}\right| \leq \frac{c|z|^{3}}{1+|z|}
$$

where $c$ denotes a generic positive constant independent of the complex number $z$. Using (3.18) in (3.16), we obtain

$$
\left\|P_{8}(k)\right\| \leq c \int_{a}^{\infty} d y\left\|\omega(0, y)^{\dagger}\right\|\|V(y)\|\left(\frac{|k| y}{1+|k y|}\right)\left(|k y|^{2}\right)
$$

and hence, due to $V \in L_{2}^{1}\left(\mathbf{R}^{+}\right)$and the boundedness of $\omega(0, y)$, which follows from (2.28) and (2.35), we obtain from (3.19) that

$$
P_{8}(k)=o\left(k^{2}\right), \quad k \rightarrow 0 \text { in } \overline{\mathbf{C}^{+}} .
$$

As for the integral in (3.14), let us first write it as

$$
P_{6}(k, x)=P_{9}\left(-k^{*}\right)^{\dagger}+o(1), \quad x \rightarrow+\infty,
$$

where we have defined

$$
P_{9}(k):=-\int_{a}^{\infty} d y e^{i k y}[\omega(k, y)-\omega(0, y)] V(y)
$$

Using (2.25) in (3.22) we get

$$
P_{9}(k)=-i k^{2} \int_{a}^{\infty} d y e^{i k y} V(y) \omega_{1}(y)-i k^{2} \int_{a}^{\infty} d y e^{i k y} V(y) \omega_{2}(k, y) .
$$

Applying (2.31), (2.33), and (2.34) in (3.23), we see that as $k \rightarrow 0$ in $\overline{\mathbf{C}^{+}}$we have

$$
P_{9}(k)=k^{2} \int_{a}^{\infty} d y\left[f(0, x)^{\dagger} f(0, x)-I_{n}\right]-k^{2} \int_{a}^{\infty} d y\left[f(0, x)-I_{n}\right]+o\left(k^{2}\right),
$$

or equivalently

$$
P_{9}\left(-k^{*}\right)^{\dagger}=k^{2} \int_{a}^{\infty} d y\left[f(0, x)^{\dagger} f(0, x)-I_{n}\right]-k^{2} \int_{a}^{\infty} d y\left[f(0, x)^{\dagger}-I_{n}\right]+o\left(k^{2}\right)
$$


Finally, using (3.12), (3.15), (3.17), (3.20), (3.21), and (3.24) in (3.5), and after using in (3.12) the expansion

$$
\begin{aligned}
i k e^{i k a} f(0, a)^{\dagger}-e^{i k a} f^{\prime}(0, a)^{\dagger}= & \left(i k-k^{2} a\right) f(0, a)^{\dagger}-\left(1+i k a-\frac{k^{2} a^{2}}{2}\right) f^{\prime}(0, a)^{\dagger} \\
& +o\left(k^{2}\right), \quad k \rightarrow 0 \text { in } \overline{\mathbf{C}^{+}},
\end{aligned}
$$

we obtain (3.3).

One important consequence of (3.3) is given in the following theorem.

Theorem 3.2 Assume that the potential $V$ satisfies (1.2) and belongs to $L_{2}^{1}\left(\mathbf{R}^{+}\right)$. Let $f(k, x)$ be the Jost solution to (1.1) appearing in (2.2). At any fixed $x \in \mathbf{R}^{+}$for which the matrix $f(0, x)$ is invertible, the matrix $f^{\prime}(k, x) f(k, x)^{-1}$ is twice differentiable at $k=0$ and we have

$$
\begin{aligned}
f^{\prime}(k, x) f(k, x)^{-1}= & f^{\prime}(0, x) f(0, x)^{-1}+i k\left[f(0, x)^{-1}\right]^{\dagger} f(0, x)^{-1} \\
& +k^{2}\left[f(0, x)^{-1}\right]^{\dagger} q_{1}(x) f(0, x)^{-1}+o\left(k^{2}\right), \quad k \rightarrow 0 \text { in } \overline{\mathbf{C}^{+}},
\end{aligned}
$$

where we have defined

$$
q_{1}(x):=-x I_{n}-i f(0, x)^{-1} \dot{f}(0, x)+\int_{x}^{\infty} d y\left[f(0, y)^{\dagger} f(0, y)-I_{n}\right] .
$$

At any $x \in \mathbf{R}^{+}$for which the matrix $f^{\prime}(0, x)$ is invertible, the matrix $f(k, x) f^{\prime}(k, x)^{-1}$ is twice differentiable at $k=0$ and we have

$$
\begin{aligned}
f(k, x) f^{\prime}(k, x)^{-1}= & f(0, x) f^{\prime}(0, x)^{-1}-i k\left[f^{\prime}(0, x)^{-1}\right]^{\dagger} f^{\prime}(0, x)^{-1} \\
& +k^{2}\left[f^{\prime}(0, x)^{-1}\right]^{\dagger} q_{2}(x) f^{\prime}(0, x)^{-1}+o\left(k^{2}\right), \quad k \rightarrow 0 \text { in } \overline{\mathbf{C}^{+}},
\end{aligned}
$$

where we have defined

$$
q_{2}(x):=x I_{n}+i f^{\prime}(0, x)^{-1} \dot{f}^{\prime}(0, x)-\int_{x}^{\infty} d y\left[f(0, y)^{\dagger} f(0, y)-I_{n}\right] .
$$

PROOF: By Proposition 2.1(a) the matrix-valued function $f(k, a)$ is continuous in $k \in \overline{\mathbf{C}^{+}}$ and hence the determinant of $f(k, a)$ is also continuous in $k \in \overline{\mathbf{C}^{+}}$. Thus, if $f(0, a)^{-1}$ exists we must have $f(k, a)$ invertible in a neighborhood of $k=0$ in $\overline{\mathbf{C}^{+}}$. Then, from (3.2) we get

$$
f^{\prime}(k, a) f(k, a)^{-1}=\left[f(0, a)^{\dagger}\right]^{-1} f^{\prime}(0, a)^{\dagger}+\left[f(0, a)^{\dagger}\right]^{-1} P(k) f(k, a)^{-1} .
$$


Note that (2.11) implies that

$$
\left[f(0, a)^{\dagger}\right]^{-1} f^{\prime}(0, a)^{\dagger}=f^{\prime}(0, a) f(0, a)^{-1}
$$

Applying (3.29) in (3.28) and using (2.12) and (3.3), we obtain (3.25). If $f^{\prime}(0, a)^{-1}$ exists, from (3.2) we get

$$
f(k, a) f^{\prime}(k, a)^{-1}=\left[f^{\prime}(0, a)^{\dagger}\right]^{-1} f(0, a)^{\dagger}-\left[f^{\prime}(0, a)^{\dagger}\right]^{-1} P(k) f^{\prime}(k, a)^{-1},
$$

and proceeding in a similar way, we obtain (3.27).

The results in (3.25) and (3.27) are remarkable because the explicit expansions are given up to $o\left(k^{2}\right)$, whereas the expansions for $f(k, x)$ and $f^{\prime}(k, x)$ given in $(2.7)$ and $(2.8)$ can only be obtained up to $o(k)$. Even though we cannot improve (2.7) or (2.8) because the existence of $\ddot{f}(0, x)$ or of $\ddot{f}^{\prime}(0, x)$ is not assured when $V \in L_{2}^{1}\left(\mathbf{R}^{+}\right)$, we are still able to have the expansions in (3.25) and (3.27) up to $o\left(k^{2}\right)$.

In the scalar case, i.e. when $n=1$ in (1.1), the selfadjointness (1.2) forces the potential $V$ to be a real-valued scalar function, and as a result $f(0, x)$ and $f^{\prime}(0, x)$ also become real valued. Then, from (3.25) and (3.27) we get the respective remarkable expansions as $k \rightarrow 0$ in $\overline{\mathbf{C}^{+}}$

$$
\begin{aligned}
\frac{f^{\prime}(k, x)}{f(k, x)}= & \frac{f^{\prime}(0, x)}{f(0, x)}+\frac{i k}{f(0, x)^{2}} \\
& +k^{2}\left[-\frac{i \dot{f}(0, x)}{f(0, x)^{3}}-\frac{x}{f(0, x)^{2}}+\frac{\int_{x}^{\infty} d y\left[f(0, y)^{2}-1\right]}{f(0, x)^{2}}\right]+o\left(k^{2}\right), \\
\frac{f(k, x)}{f^{\prime}(k, x)}= & \frac{f(0, x)}{f^{\prime}(0, x)}-\frac{i k}{f^{\prime}(0, x)^{2}} \\
& +k^{2}\left[\frac{i \dot{f}^{\prime}(0, x)}{f^{\prime}(0, x)^{3}}+\frac{x}{f^{\prime}(0, x)^{2}}-\frac{\int_{x}^{\infty} d y\left[f(0, y)^{2}-1\right]}{f^{\prime}(0, x)^{2}}\right]+o\left(k^{2}\right) .
\end{aligned}
$$

We recall that, in the scalar case, $f(0, x)$ and $f^{\prime}(0, x)$ cannot simultaneously be zero at the same $x$-value because that would imply that a corresponding initial-value problem for (2.5) on the interval $(x,+\infty)$ would only have the zero solution $f(0, x) \equiv 0$, contradicting (2.6). 


\section{THE JOST MATRIX AND THE SCATTERING MATRIX}

In this section we recall the definition of the Jost matrix and the scattering matrix for (1.1) with a selfadjoint matrix potential $V$ in $L_{2}^{1}\left(\mathbf{R}^{+}\right)$and with the selfadjoint boundary condition (1.3).

The Jost matrix $J(k)$ for $k \in \overline{\mathbf{C}^{+}}$is defined $[5,17,21]$ as

$$
J(k):=\left[f\left(-k^{*}, x\right)^{\dagger} ; \varphi(k, x)\right],
$$

where $f(k, x)$ is the Jost solution to (1.1) appearing in $(2.2)$ and $\varphi(k, x)$ is the $n \times n$ matrixvalued solution to (1.1) appearing in (2.45). Since the Wronskian in (4.1) is independent of $x$, its evaluation at $x=0$ and $(2.45)$ yield

$$
J(k)=f\left(-k^{*}, 0\right)^{\dagger} B-f^{\prime}\left(-k^{*}, 0\right)^{\dagger} A,
$$

where $A$ and $B$ are the matrices appearing in (1.3) and (1.4). Note that $J(k)$ is not uniquely determined by the potential $V$ and the selfadjoint boundary condition (1.3). This is because $J(k)$ is unique up to $[5,17]$ a right multiplication by a constant invertible matrix, i.e. by a constant matrix not depending on $k$. It is known $[5,17]$ that $J(k)$ is invertible for $k \in \mathbf{R} \backslash\{0\}$ even under the weaker assumption $V \in L_{1}^{1}\left(\mathbf{R}^{+}\right)$.

The scattering matrix $S(k)$ is defined as [5,16-18]

$$
S(k):=-J(-k) J(k)^{-1}, \quad k \in \mathbf{R} \backslash\{0\},
$$

and it is uniquely determined by the boundary condition and the potential $V$. Even though $J(k)$ is uniquely defined only up to a right multiplication by a constant invertible matrix, the unique determination of $S(k)$ is assured because $S(k)$ remains unchanged when $J(k)$ is multiplied on the right by a constant invertible matrix. We have already established [5] the continuity of $S(k)$ at $k=0$ when the potential satisfies (1.2) and $V \in L_{1}^{1}\left(\mathbf{R}^{+}\right)$. One of our primary goals in the current paper is to establish the differentiability of $S(k)$ at $k=0$ under the further assumption $V \in L_{2}^{1}\left(\mathbf{R}^{+}\right)$. 


\section{SMALL- $k$ BEHAVIOR IN THE GENERIC CASE}

When the potential in (1.1) is selfadjoint and belongs to $L_{2}^{1}\left(\mathbf{R}^{+}\right)$, we are interested in analyzing the behavior of $J(k)$ and $J(k)^{-1}$ in a neighborhood of $k=0$ in $\overline{\mathbf{C}^{+}}$and the behavior of $S(k)$ in a neighborhood of $k=0$ in $\mathbf{R}$. There are two cases to consider. The first is the generic case where $J(0)$ is invertible and the other is the exceptional case where $J(0)$ is not invertible. The analysis in the exceptional case is delicate, and it will be given in Section 7. In this section we provide the analysis in the generic case. We prove the differentiability at $k=0$ for $J(k), J(k)^{-1}$, and $S(k)$, and we also provide their explicit small- $k$ expansions up to $o(k)$ as $k \rightarrow 0$.

Theorem 5.1 Assume that the potential $V$ is selfadjoint and belongs to $L_{2}^{1}\left(\mathbf{R}^{+}\right)$, and let $f(k, x)$ be the Jost solution appearing in (2.2) and $J(k)$ be the associated Jost matrix defined in (4.1). Then:

(a) The Jost matrix is differentiable at $k=0$ in $\overline{\mathbf{C}^{+}}$and we have

$$
J(k)=J(0)+k \dot{J}(0)+o(k), \quad k \rightarrow 0 \text { in } \overline{\mathbf{C}^{+}},
$$

where

$$
\begin{aligned}
& J(0)=f(0,0)^{\dagger} B-f^{\prime}(0,0)^{\dagger} A, \\
& \dot{J}(0)=\dot{f}^{\prime}(0,0)^{\dagger} A-\dot{f}(0,0)^{\dagger} B,
\end{aligned}
$$

with $A$ and $B$ being the constant matrices appearing in (1.3).

(b) If $J(0)$ is invertible, then the inverse of the Jost matrix is differentiable at $k=0$ in $\overline{\mathbf{C}^{+}}$and we have

$$
J(k)^{-1}=J(0)^{-1}-k J(0)^{-1} \dot{J}(0) J(0)^{-1}+o(k), \quad k \rightarrow 0 \text { in } \overline{\mathbf{C}^{+}},
$$

with $J(0)$ and $\dot{J}(0)$ given as in (5.2) and (5.3), respectively.

(c) If $J(0)$ is invertible, then the scattering matrix $S(k)$ is differentiable at $k=0$ in $\mathbf{R}$ and we have

$$
S(k)=-I_{n}+2 k \dot{J}(0) J(0)^{-1}+o(k), \quad k \rightarrow 0 \text { in } \mathbf{R},
$$


with $J(0)$ and $\dot{J}(0)$ given as in (5.2) and (5.3), respectively.

PROOF: Using (2.7) and (2.8) in (4.2) we obtain (5.1). If $J(0)$ is invertible, we must have $\operatorname{det}[J(0)] \neq 0$. Because of the continuity $[5]$ of $J(k)$ in $\overline{\mathbf{C}^{+}}$, the matrix $J(k)^{-1}$ exists in a neighborhood of $k=0$ in $\overline{\mathbf{C}^{+}}$if it exists at $k=0$. We obtain (5.4) from (5.1) by using

$$
J(k)^{-1}=\left[I_{n}+k J(0)^{-1} \dot{J}(0)+o(k)\right]^{-1} J(0)^{-1}, \quad k \rightarrow 0 \text { in } \overline{\mathbf{C}^{+}} .
$$

Using (5.1) and (5.4) in (4.3) we establish (5.5).

Let us emphasize that Theorem 5.1(a) holds both in the generic and exceptional cases because the invertibility of $J(0)$ is not needed there. We also note that the coefficients in the expansions of (5.1), (5.4), and (5.5) can be constructed from knowledge of the potential $V$ and the constant matrices $A$ and $B$. This is because $f(0, x)$ and $\dot{f}(0, x)$ can be obtained by solving (2.5) with the respective asymptotic conditions (2.14) and (2.18), and hence we directly obtain the constant matrices $f(0,0)$ and $\dot{f}(0,0)$, and through differentiation, the constant matrices $f^{\prime}(0,0)$ and $\dot{f}^{\prime}(0,0)$. Thus, as seen from (5.2) and (5.3), we have all the ingredients to determine the coefficients in the expansions of (5.1), (5.4), and (5.5).

\section{INVERTIBILITY OF A RELATED MATRIX}

In this section we continue to analyze the small- $k$ behavior of the Jost matrix $J(k)$ defined in (4.1). Let us recall that the $n \times n$ matrix $J(0)$ is invertible in the generic case but not invertible in the exceptional case. We show in Theorem 6.1 that the related $2 n \times 2 n$ matrix $\mathcal{J}$ defined in (6.3) is always invertible whether we are in the generic case or in the exceptional case.

Let us use $K(k)$ to denote the Jost matrix for (1.1) when the constant matrices $A$ and $B$ appearing in (1.3) are replaced with $-B$ and $A$, respectively. With the help of (5.2) and (5.3), we have

$$
\begin{gathered}
K(0)=f(0,0)^{\dagger} A+f^{\prime}(0,0)^{\dagger} B, \\
\dot{K}(0)=-\dot{f}(0,0)^{\dagger} A-\dot{f}^{\prime}(0,0)^{\dagger} B .
\end{gathered}
$$


Let us define the $2 n \times 2 n$ matrix $\mathcal{J}$ as

$$
\mathcal{J}:=\left[\begin{array}{cc}
J(0) & K(0) \\
\dot{J}(0) & \dot{K}(0)
\end{array}\right]
$$

The following result shows that $\mathcal{J}$ is always invertible even when $J(0)$ is not invertible.

Theorem 6.1 Assume that the potential $V$ is selfadjoint and belongs to $L_{2}^{1}\left(\mathbf{R}^{+}\right)$. Then, the matrix $\mathcal{J}$ defined in (6.3) is always invertible and $\mathcal{J}^{-1}$ is given by

$$
\mathcal{J}^{-1}=\left[\begin{array}{cc}
i\left(A^{\dagger} A+B^{\dagger} B\right)^{-1} \dot{K}(0)^{\dagger} & i\left(A^{\dagger} A+B^{\dagger} B\right)^{-1} K(0)^{\dagger} \\
-i\left(A^{\dagger} A+B^{\dagger} B\right)^{-1} \dot{J}(0)^{\dagger} & -i\left(A^{\dagger} A+B^{\dagger} B\right)^{-1} J(0)^{\dagger}
\end{array}\right] .
$$

PROOF: With the help of (5.2), (5.3), (6.1), and (6.2), we see that

$$
\mathcal{J}=\left[\begin{array}{cc}
I_{n} & 0 \\
0 & -I_{n}
\end{array}\right]\left[\begin{array}{ll}
f(0,0) & \dot{f}(0,0) \\
f^{\prime}(0,0) & \dot{f}^{\prime}(0,0)
\end{array}\right]^{\dagger}\left[\begin{array}{cc}
I_{n} & 0 \\
0 & -I_{n}
\end{array}\right]\left[\begin{array}{cc}
B & A \\
A & -B
\end{array}\right]
$$

Each matrix on the right-hand side in (6.5) is invertible. The invertibility of the second matrix on the right-hand side in (6.5) is assured by (2.19). From (1.15) and (1.16) of [5] we have

$$
\left[\begin{array}{cc}
B & A \\
A & -B
\end{array}\right]^{-1}=\left[\begin{array}{cc}
\left(A^{\dagger} A+B^{\dagger} B\right)^{-1} & 0 \\
0 & \left(A^{\dagger} A+B^{\dagger} B\right)^{-1}
\end{array}\right]\left[\begin{array}{cc}
B & A \\
A & -B
\end{array}\right]^{\dagger}
$$

where we recall that $A^{\dagger} A+B^{\dagger} B$ is positive and hence invertible. Thus, with the help of (2.19) and (6.6), from (6.5) we get

$$
\mathcal{J}^{-1}=-i\left[\begin{array}{cc}
\left(A^{\dagger} A+B^{\dagger} B\right)^{-1} & 0 \\
0 & \left(A^{\dagger} A+B^{\dagger} B\right)^{-1}
\end{array}\right]\left[\begin{array}{cc}
B^{\dagger} & A^{\dagger} \\
A^{\dagger} & -B^{\dagger}
\end{array}\right]\left[\begin{array}{cc}
\dot{f}^{\prime}(0,0) & -f^{\prime}(0,0) \\
\dot{f}(0,0) & -f(0,0)
\end{array}\right],
$$

which, by using (5.2), (5.3), (6.1), and (6.2), can also be written as (6.4).

\section{SMALL- $k$ ANALYSIS OF $J(k)^{-1}$ AND $S(k)$ IN THE EXCEPTIONAL CASE}

In the exceptional case $J(0)$ is not invertible, and hence we cannot use (5.4) to express the behavior of $J(k)^{-1}$ as $k \rightarrow 0$ in $\overline{\mathbf{C}^{+}}$and we cannot use (5.5) to express the behavior of 
$S(k)$ as $k \rightarrow 0$ in $\mathbf{R}$. Our goal in this section is to obtain such behaviors in the exceptional case when the potential $V$ in (1.1) satisfies (1.2) and belongs to $V \in L_{2}^{1}\left(\mathbf{R}^{+}\right)$. We analyzed such behaviors in [5] under the weaker assumption $V \in L_{1}^{1}\left(\mathbf{R}^{+}\right)$instead of $V \in L_{2}^{1}\left(\mathbf{R}^{+}\right)$ and proved that $J(k)^{-1}$ has a simple pole at $k=0$ but $S(k)$ is continuous at $k=0$. In the current paper, under the stronger assumption $V \in L_{2}^{1}\left(\mathbf{R}^{+}\right)$, we sharpen the small- $k$ estimates and also prove that $S(k)$ is differentiable at $k=0$. Our main results are given in Theorem 7.6, where we prove that $J(k), k J(k)^{-1}$, and $S(k)$ are differentiable at $k=0$, and in their expansions as $k \rightarrow 0$ we provide the explicit coefficients up to $o(k)$.

The following result is already known and the proof is given in Proposition 5.4 of [5]. Proposition 7.1 Assume that $V$ in (1.1) is selfadjoint and belongs to $L_{1}^{1}\left(\mathbf{R}^{+}\right)$. Let $f(k, x)$, $\omega(k, x)$, and $\varphi(k, x)$ be the solutions to (1.1) appearing in (2.2), (2.21), and (2.45), respectively. Then, the Jost matrix defined in (4.1) can be written as

$$
J(k)=T_{1}(k)+T_{2}(k), \quad k \in \overline{\mathbf{C}^{+}},
$$

where we have defined

$$
\begin{gathered}
T_{1}(k):=-P\left(-k^{*}\right)^{\dagger} f(0, a)^{-1} \varphi(k, a), \\
T_{2}(k):=f\left(-k^{*}, a\right)^{\dagger}\left[f(0, a)^{-1}\right]^{\dagger}\left[\omega\left(-k^{*}, x\right)^{\dagger} ; \varphi(k, x)\right],
\end{gathered}
$$

with $P(k)$ being the matrix defined in (3.1) and a being the nonnegative constant appearing in (2.21).

The small- $k$ behavior of the Wronskian $\left[\omega\left(-k^{*}, x\right)^{\dagger} ; \varphi(k, x)\right]$ appearing in $(7.3)$ is analyzed in the next proposition when $V \in L_{2}^{1}\left(\mathbf{R}^{+}\right)$.

Proposition 7.2 Assume that $V$ in (1.1) is selfadjoint and belongs to $L_{2}^{1}\left(\mathbf{R}^{+}\right)$. Then, the Wronskian appearing in (7.3) has the small-k asymptotics

$$
\left[\omega\left(-k^{*}, x\right)^{\dagger} ; \varphi(k, x)\right]=J(0)+i k^{2}\left[\omega_{1}^{\prime}(0)^{\dagger} A-\omega_{1}(0)^{\dagger} B\right]+o\left(k^{2}\right), \quad k \rightarrow 0 \text { in } \overline{\mathbf{C}^{+}},
$$


where $A$ and $B$ are the matrices appearing in (1.3), $J(k)$ is the Jost matrix defined in (4.1), and $\omega_{1}(x)$ is the matrix defined in (2.26).

PROOF: Since the value of our Wronskian is independent of $x$, we can evaluate its value at $x=0$. By writing

$$
\left[\omega\left(-k^{*}, x\right)^{\dagger} ; \varphi(k, x)\right]=\left[\omega(0, x)^{\dagger} ; \varphi(k, x)\right]+\left[\omega\left(-k^{*}, x\right)^{\dagger}-\omega(0, x)^{\dagger} ; \varphi(k, x)\right],
$$

from $(2.21),(2.28),(2.45)$, and (4.2) we see that the first Wronskian on the right-hand side in (7.5), when $x=0$, yields

$$
\left.\left[\omega(0, x)^{\dagger} ; \varphi(k, x)\right]\right|_{x=0}=J(0) .
$$

Next, we evaluate at $x=0$ the value of the second Wronskian on the right-hand side in (7.5). From (2.27) we see that

$$
\omega_{2}(0, x)=0, \quad \omega_{2}^{\prime}(0, x)=0, \quad x \in \mathbf{R}^{+},
$$

and hence $(2.25)$ yields

$$
\omega(k, x)-\omega(0, x)=i k^{2} \omega_{1}(x)+o\left(k^{2}\right), \quad k \rightarrow 0 \text { in } \overline{\mathbf{C}^{+}} .
$$

Using (2.46) and (7.7) with $x=0$ in the second Wronskian on the right-hand side of (7.5), we get

$$
\left[\omega\left(-k^{*}, x\right)^{\dagger}-\omega(0, x)^{\dagger} ; \varphi(k, x)\right]_{x=0}=i k^{2}\left[\omega_{1}^{\prime}(0)^{\dagger} A-\omega_{1}(0)^{\dagger} B\right]+o\left(k^{2}\right), \quad k \rightarrow 0 \text { in } \overline{\mathbf{C}^{+}}
$$

and hence (7.6) and (7.8) yield (7.4).

In order to analyze the small- $k$ behavior of $J(k)$, from (7.1), (7.3), and (7.5) we see that it is convenient to analyze the small- $k$ behavior of the related matrix $F(k)$ defined as

$$
F(k):=f(0, a)^{\dagger}\left[f\left(-k^{*}, a\right)^{\dagger}\right]^{-1} J(k) .
$$


Proposition 7.3 Assume that $V$ in (1.1) is selfadjoint and belongs to $L_{2}^{1}\left(\mathbf{R}^{+}\right)$. Then, the $n \times n$ matrix $F(k)$ defined in (7.9) has the small-k asymptotics

$$
F(k)=J(0)-i k \mathcal{R}+k^{2} F_{2}+o\left(k^{2}\right), \quad k \rightarrow 0 \text { in } \overline{\mathbf{C}^{+}},
$$

where we have defined

$$
\begin{gathered}
\mathcal{R}:=f(0, a)^{-1} \varphi(0, a), \\
F_{2}:=i\left[\omega_{1}^{\prime}(0)^{\dagger} A-\omega_{1}(0)^{\dagger} B\right]-q_{1}(a)^{\dagger} \mathcal{R},
\end{gathered}
$$

with $f(k, x)$ and $\varphi(k, x)$ being the solutions to (1.1) and satisfying (2.2) and (2.45), respectively, and where $\omega_{1}(x)$ and $q_{1}(x)$ are the quantities defined in (2.26) and (3.26), respectively, and $a$ is the nonnegative constant appearing in (2.21).

PROOF: From (7.1)-(7.3) we see that

$$
F(k)=-f(0, a)^{\dagger}\left[f\left(-k^{*}, a\right)^{\dagger}\right]^{-1} P\left(-k^{*}\right)^{\dagger} f(0, a)^{-1} \varphi(k, a)+\left[\omega\left(-k^{*}, x\right)^{\dagger} ; \varphi(k, x)\right] .
$$

Using (2.12), (2.46), (3.3), and (7.4) in (7.13), we get the expansion in (7.10).

Since $J(0)$ is not invertible in the exceptional case, in order to analyze the small- $k$ behavior of $J(k)^{-1}$, we will transform (7.10) into the representation where the matrix $J(0)$ is in its Jordan canonical form with the zero eigenvalues appearing in the upper blocks. We refer the reader to Section VI of [5], and in particular to (6.7) of [5], where such a similarity transformation is accomplished by the invertible constant matrix $\mathcal{S}$ constructed with the help of the eigenvectors and generalized eigenvectors associated with the zero eigenvalue of the matrices $J(0)$ and $J(0)^{\dagger}$.

As in (6.12) of [5], let us introduce

$$
\mathcal{Z}(k):=\left[\begin{array}{ll}
\mathcal{A}(k) & \mathcal{B}(k) \\
\mathcal{C}(k) & \mathcal{D}(k)
\end{array}\right]:=P_{2} \mathcal{S}^{-1} F(k) \mathcal{S} P_{1},
$$

where $\mathcal{A}(k)$ has size $\mu \times \mu$ and $\mathcal{D}(k)$ has size $(n-\mu) \times(n-\mu)$, and $P_{1}$ and $P_{2}$ are the permutation matrices defined in (6.11) of [5]. Here, $\mu$ is the geometric multiplicity of the 
zero eigenvalue of $J(0)$. The matrices $P_{1}$ and $P_{2}$ help to move the elements 1 appearing in the Jordan canonical form of $J(0)$ from the superdiagonal to the diagonal so that the Jordan block form of $J(0)$ is transformed into the form given in (7.17), where $P_{1}$ permutes the rows and $P_{2}$ permutes the columns of the Jordan canonical form of $J(0)$.

Proposition 7.4 Assume that $V$ in (1.1) is selfadjoint and belongs to $L_{2}^{1}\left(\mathbf{R}^{+}\right)$. Then:

(a) The small-k asymptotics as $k \rightarrow 0$ in $\overline{\mathbf{C}^{+}}$of the $n \times n$ matrix $\mathcal{Z}(k)$ defined in (7.14) is given by

$$
\mathcal{Z}(k)=P_{2} \mathcal{S}^{-1} J(0) \mathcal{S} P_{1}-i k P_{2} \mathcal{S}^{-1} \mathcal{R} \mathcal{S} P_{1}+k^{2} P_{2} \mathcal{S}^{-1} F_{2} \mathcal{S} P_{1}+o\left(k^{2}\right)
$$

where $\mathcal{R}$ and $F_{2}$ are the constant $n \times n$ matrices defined in (7.11) and (7.12), respectively.

(b) In the exceptional case, i.e. when $J(0)$ is not invertible, the expansion in (7.15) is equivalent to the expansion as $k \rightarrow 0$ in $\overline{\mathbf{C}^{+}}$

$$
\left[\begin{array}{ll}
\mathcal{A}(k) & \mathcal{B}(k) \\
\mathcal{C}(k) & \mathcal{D}(k)
\end{array}\right]=\left[\begin{array}{cc}
k \mathcal{A}_{1}+k^{2} \mathcal{A}_{2}+o\left(k^{2}\right) & k \mathcal{B}_{1}+k^{2} \mathcal{B}_{2}+o\left(k^{2}\right) \\
k \mathcal{C}_{1}+k^{2} \mathcal{C}_{2}+o\left(k^{2}\right) & \mathcal{D}_{0}+k \mathcal{D}_{1}+k^{2} \mathcal{D}_{2}+o\left(k^{2}\right)
\end{array}\right]
$$

with $\mathcal{A}_{1}$ and $\mathcal{D}_{0}$ being constant invertible matrices of sizes $\mu \times \mu$ and $(n-\mu) \times(n-\mu)$, respectively, and where $\mathcal{A}_{2}, \mathcal{B}_{1}, \mathcal{B}_{2}, \mathcal{C}_{1}, \mathcal{C}_{2}, \mathcal{D}_{1}$, and $\mathcal{D}_{2}$ are some constant matrices.

PROOF: We obtain (7.15) directly from (7.10) by multiplying on the left and on the right with the constant matrices $P_{2} \mathcal{S}^{-1}$ and $\mathcal{S} P_{1}$, respectively. We refer the reader to Section VI of [5] for the expansion in (7.15) without the term containing $k^{2}$ when the potential satisfies the weaker assumption $V \in L_{1}^{1}\left(\mathbf{R}^{+}\right)$. In the expansion in (7.15), the constant term $P_{2} \mathcal{S}^{-1} J(0) \mathcal{S} P_{1}$ has the form given in the displayed formula before (6.1) of [5], namely

$$
P_{2} \mathcal{S}^{-1} J(0) \mathcal{S} P_{1}=\operatorname{diag}\left\{0_{\mu}, I_{\nu-\mu}, J_{n_{\mu+1}}\left(\lambda_{\mu+1}\right), \ldots, J_{n_{\kappa}}\left(\lambda_{\kappa}\right)\right\}
$$

In the block diagonal matrix given on the right-hand side of (7.17) the zero block matrix $0_{\mu}$ has size $\mu \times \mu$, where we recall that $\mu$ is the geometric multiplicity of the zero eigenvalue 
of $J(0)$. The identity matrix $I_{\nu-\mu}$ in (7.17) has size $(\nu-\mu) \times(\nu-\mu)$, where $\nu$ denotes the algebraic multiplicity of the zero eigenvalue of $J(0)$. The remaining block matrices $J_{n_{\alpha}}\left(\lambda_{\alpha}\right)$ for $\alpha=\mu+1, \ldots, \kappa$ are the Jordan blocks associated with the nonzero eigenvalues of $J(0)$, as described in (6.7) of [5]. Let us use $\mathcal{D}_{0}$ to denote the nonzero block diagonal matrix of size $(n-\mu) \times(n-\mu)$ in $(7.17)$, i.e.

$$
\mathcal{D}_{0}:=\operatorname{diag}\left\{I_{\nu-\mu}, J_{n_{\mu+1}}\left(\lambda_{\mu+1}\right), \ldots, J_{n_{\kappa}}\left(\lambda_{\kappa}\right)\right\}
$$

Since none of the eigenvalues $\lambda_{\alpha}$ for $\alpha=\mu+1, \ldots, \kappa$ appearing in (7.18) are zero, the matrix $\mathcal{D}_{0}$ is invertible. The term proportional to $k$ in (7.16) is already known and has been analyzed in Theorem 6.1 of [5] under the weaker assumption $V \in L_{1}^{1}\left(\mathbf{R}^{+}\right)$. As in (6.13) of [5], we have

$$
\left[\begin{array}{ll}
\mathcal{A}_{1} & \mathcal{B}_{1} \\
\mathcal{C}_{1} & \mathcal{D}_{1}
\end{array}\right]:=-i P_{2} \mathcal{S}^{-1} \mathcal{R} \mathcal{S} P_{1},
$$

where $\mathcal{A}_{1}$ is invertible, which is proved in Theorem 6.1 of [5]. For the term proportional to $k^{2}$ in the expansion of (7.17), let us introduce the block matrix notation

$$
\left[\begin{array}{ll}
\mathcal{A}_{2} & \mathcal{B}_{2} \\
\mathcal{C}_{2} & \mathcal{D}_{2}
\end{array}\right]:=P_{2} \mathcal{S}^{-1} F_{2} \mathcal{S} P_{1},
$$

where $\mathcal{A}_{2}$ has size $\mu \times \mu$ and $\mathcal{D}_{2}$ has size $(n-\mu) \times(n-\mu)$ and hence $\mathcal{B}_{2}$ and $\mathcal{C}_{2}$ have sizes $\mu \times(n-\mu)$ and $(n-\mu) \times \mu$, respectively. Using (7.14) and (7.17)-(7.20) in (7.15) we obtain (7.16).

Next, we analyze the small- $k$ asymptotics for the inverse of the matrix $\mathcal{Z}(k)$ defined in (7.14).

Proposition 7.5 Assume that the potential $V$ in (1.1) is selfadjoint and belongs to $L_{2}^{1}\left(\mathbf{R}^{+}\right)$, and let $\mathcal{Z}(k)$ be the $n \times n$ matrix defined in (7.14). In the exceptional case, i.e. when $J(0)$ is not invertible, $\mathcal{Z}(k)^{-1}$ has the small-k asymptotics as $k \rightarrow 0$ in $\overline{\mathbf{C}^{+}}$

$$
\mathcal{Z}(k)^{-1}=\left[\begin{array}{cc}
\frac{1}{k} \mathcal{A}_{1}^{-1}+\mathcal{Y}_{1}+o(1) & -\mathcal{A}_{1}^{-1} \mathcal{B}_{1} \mathcal{D}_{0}^{-1}+k \mathcal{Y}_{2}+o(k) \\
-\mathcal{D}_{0}^{-1} \mathcal{C}_{1} \mathcal{A}_{1}^{-1}+k \mathcal{Y}_{3}+o(k) & \mathcal{D}_{0}^{-1}+k \mathcal{Y}_{4}+O\left(k^{2}\right)
\end{array}\right],
$$


where we have defined, in terms of the matrices appearing in (7.18)-(7.20),

$$
\begin{gathered}
\mathcal{Y}_{1}:=\mathcal{A}_{1}^{-1}\left(\mathcal{B}_{1} \mathcal{D}_{0}^{-1} \mathcal{C}_{1}-\mathcal{A}_{2}\right) \mathcal{A}_{1}^{-1} \\
\mathcal{Y}_{2}:=\mathcal{A}_{1}^{-1}\left[\mathcal{A}_{2} \mathcal{A}_{1}^{-1} \mathcal{B}_{1}-\mathcal{B}_{1} \mathcal{D}_{0}^{-1} \mathcal{C}_{1} \mathcal{A}_{1}^{-1} \mathcal{B}_{1}-\mathcal{B}_{2}-\mathcal{B}_{1} \mathcal{D}_{0}^{-1} \mathcal{D}_{1}\right] \mathcal{D}_{0}^{-1} \\
\mathcal{Y}_{3}:=\mathcal{D}_{0}^{-1}\left[\mathcal{D}_{1} \mathcal{D}_{0}^{-1} \mathcal{C}_{1}-\mathcal{C}_{2}+\mathcal{C}_{1} \mathcal{A}_{1}^{-1} \mathcal{A}_{2}-\mathcal{C}_{1} \mathcal{A}_{1}^{-1} \mathcal{B}_{1} \mathcal{D}_{0}^{-1} \mathcal{C}_{1}\right] \mathcal{A}_{1}^{-1} \\
\mathcal{Y}_{4}:=\mathcal{D}_{0}^{-1}\left(\mathcal{C}_{1} \mathcal{A}_{1}^{-1} \mathcal{B}_{1}-\mathcal{D}_{1}\right) \mathcal{D}_{0}^{-1}
\end{gathered}
$$

PROOF: We can readily evaluate the inverse of $\mathcal{Z}(k)$ appearing in (7.14) by using (6.16) of [5], namely

$$
\left[\begin{array}{cc}
\mathcal{A} & \mathcal{B} \\
\mathcal{C} & \mathcal{D}
\end{array}\right]^{-1}=\left[\begin{array}{cc}
\left(\mathcal{A}-\mathcal{B D}^{-1} \mathcal{C}\right)^{-1} & -\left(\mathcal{A}-\mathcal{B D}^{-1} \mathcal{C}\right)^{-1} \mathcal{B D}^{-1} \\
-\mathcal{D}^{-1} \mathcal{C}\left(\mathcal{A}-\mathcal{B D} \mathcal{D}^{-1} \mathcal{C}\right)^{-1} & \mathcal{D}^{-1} \mathcal{C}\left(\mathcal{A}-\mathcal{B D}^{-1} \mathcal{C}\right)^{-1} \mathcal{B D}^{-1}+\mathcal{D}^{-1}
\end{array}\right]
$$

as well as the expansions as $k \rightarrow 0$ in $\overline{\mathbf{C}^{+}}$

$$
\begin{gathered}
{\left[k \mathcal{A}_{1}+k^{2} \mathcal{A}_{2}+o\left(k^{2}\right)\right]^{-1}=\frac{1}{k} \mathcal{A}_{1}^{-1}-\mathcal{A}_{1}^{-1} \mathcal{A}_{2} \mathcal{A}_{1}^{-1}+o(1),} \\
{\left[\mathcal{D}_{0}+k \mathcal{D}_{1}+k^{2} \mathcal{D}_{2}+o\left(k^{2}\right)\right]^{-1}=} \\
\mathcal{D}_{0}^{-1}-k \mathcal{D}_{0}^{-1} \mathcal{D}_{1} \mathcal{D}_{0}^{-1} \\
\quad+k^{2}\left[\mathcal{D}_{0}^{-1} \mathcal{D}_{1} \mathcal{D}_{0}^{-1} \mathcal{D}_{1} \mathcal{D}_{0}^{-1}-\mathcal{D}_{0}^{-1} \mathcal{D}_{2} \mathcal{D}_{0}^{-1}\right]+o\left(k^{2}\right),
\end{gathered}
$$

where we recall that the invertibility of $\mathcal{A}_{1}$ and $\mathcal{D}_{0}$ is already known [5]. Using (7.16), (7.24), and an analog of (7.23) on the right-hand side of (7.22), we obtain (7.21).

We are now ready to evaluate the small- $k$ asymptotics of the Jost matrix $J(k)$ defined in (4.1), its inverse $J(k)^{-1}$, and the scattering matrix $S(k)$ defined in (4.3). From (7.9) and (7.14) we see that the Jost matrix $J(k)$ is given by

$$
J(k)=f\left(-k^{*}, a\right)^{\dagger}\left[f(0, a)^{\dagger}\right]^{-1} \mathcal{S} P_{2}^{-1} \mathcal{Z}(k) P_{1}^{-1} \mathcal{S}^{-1},
$$

where the small- $k$ asymptotics in $\overline{\mathbf{C}^{+}}$will be evaluated with the help of (7.16). On the other hand, from (7.25) we get

$$
J(k)^{-1}=\mathcal{S} P_{1} \mathcal{Z}(k)^{-1} P_{2} \mathcal{S}^{-1} f(0, a)^{\dagger}\left[f\left(-k^{*}, a\right)^{\dagger}\right]^{-1},
$$


where the small- $k$ asymptotics in $\overline{\mathbf{C}^{+}}$will be evaluated with the help of (7.21). Using (7.25) and (7.26) in (4.3) we obtain for $k \in \mathbf{R} \backslash\{0\}$

$$
S(k)=-f(k, a)^{\dagger}\left[f(0, a)^{\dagger}\right]^{-1} \mathcal{S} P_{2}^{-1} \mathcal{Z}(-k) \mathcal{Z}(k)^{-1} P_{2} \mathcal{S}^{-1} f(0, a)^{\dagger}\left[f(-k, a)^{\dagger}\right]^{-1},
$$

where the small- $k$ asymptotics will be evaluated with the help of (7.16) and (7.21). In evaluating the small- $k$ asymptotics of $J(k), J(k)^{-1}$, and $S(k)$, we will also use some expansions related to (2.7) and (2.12), namely

$$
\begin{aligned}
& f\left(-k^{*}, a\right)^{\dagger}\left[f(0, a)^{\dagger}\right]^{-1}=I_{n}-k \dot{f}(0, a)^{\dagger}\left[f(0, a)^{\dagger}\right]^{-1}+o(k), \quad k \rightarrow 0 \text { in } \overline{\mathbf{C}^{+}} \\
& f(0, a)^{\dagger}\left[f\left(-k^{*}, a\right)^{\dagger}\right]^{-1}=I_{n}+k \dot{f}(0, a)^{\dagger}\left[f(0, a)^{\dagger}\right]^{-1}+o(k), \quad k \rightarrow 0 \text { in } \overline{\mathbf{C}^{+}}
\end{aligned}
$$

Theorem 7.6 Assume that $V$ in (1.1) is selfadjoint and belongs to $L_{2}^{1}\left(\mathbf{R}^{+}\right)$. Let $J(k)$ be the Jost matrix defined in (4.1) and assume that we are in the exceptional case, i.e. J(0) is not invertible. Then:

(a) The Jost matrix $J(k)$ is differentiable at $k=0$ in $\overline{\mathbf{C}^{+}}$and has the behavior

$$
J(k)=J(0)+k \dot{J}(0)+o(k), \quad k \rightarrow 0 \text { in } \overline{\mathbf{C}^{+}},
$$

where $J(0)$ and $\dot{J}(0)$ are given, in terms of the matrices appearing in (7.17)-(7.19), by

$$
\begin{gathered}
J(0)=\mathcal{S} P_{2}^{-1}\left[\begin{array}{cc}
0 & 0 \\
0 & \mathcal{D}_{0}
\end{array}\right] P_{1}^{-1} \mathcal{S}^{-1} \\
\dot{J}(0)=-\dot{f}(0, a)^{\dagger}\left[f(0, a)^{\dagger}\right]^{-1} J(0)+\mathcal{S} P_{2}^{-1}\left[\begin{array}{cc}
\mathcal{A}_{1} & \mathcal{B}_{1} \\
\mathcal{C}_{1} & \mathcal{D}_{1}
\end{array}\right] P_{1}^{-1} \mathcal{S}^{-1} .
\end{gathered}
$$

(b) The Jost matrix $J(k)$ has a simple pole at $k=0$, and we have the asymptotics

$$
J(k)^{-1}=\frac{1}{k} \mathcal{S} P_{1}\left[\begin{array}{rr}
\mathcal{A}_{1}^{-1} & 0 \\
0 & 0
\end{array}\right] P_{2} \mathcal{S}^{-1}+\mathcal{E}_{1}+o(1), \quad k \rightarrow 0 \text { in } \overline{\mathbf{C}^{+}},
$$

where we have defined

$$
\begin{aligned}
\mathcal{E}_{1}:= & \mathcal{S} P_{1}\left[\begin{array}{rr}
\mathcal{A}_{1}^{-1} & 0 \\
0 & 0
\end{array}\right] P_{2} \mathcal{S}^{-1} \dot{f}(0, a)^{\dagger}\left[f(0, a)^{\dagger}\right]^{-1} \\
& +\mathcal{S} P_{1}\left[\begin{array}{cc}
\mathcal{A}_{1}^{-1}\left(\mathcal{B}_{1} \mathcal{D}_{0}^{-1} \mathcal{C}_{1}-\mathcal{A}_{2}\right) \mathcal{A}_{1}^{-1} & -\mathcal{A}_{1}^{-1} \mathcal{B}_{1} \mathcal{D}_{0}^{-1} \\
-\mathcal{D}_{0}^{-1} \mathcal{C}_{1} \mathcal{A}_{1}^{-1} & \mathcal{D}_{0}^{-1}
\end{array}\right] P_{2} \mathcal{S}^{-1} .
\end{aligned}
$$


(c) The scattering matrix $S(k)$ defined in (4.3) is differentiable at $k=0$ in $\mathbf{R}$ and satisfies the asymptotics

$$
S(k)=S(0)+k \dot{S}(0)+o(k), \quad k \rightarrow 0 \text { in } \mathbf{R},
$$

where we have

$$
\begin{gathered}
S(0)=\mathcal{S} P_{2}^{-1}\left[\begin{array}{cc}
I_{\mu} & 0 \\
2 \mathcal{C}_{1} \mathcal{A}_{1}^{-1} & -I_{n-\mu}
\end{array}\right] P_{2} \mathcal{S}^{-1} \\
\dot{S}(0)=\mathcal{S} P_{2}^{-1} \mathcal{E}_{2} P_{2} \mathcal{S}^{-1}+S(0) \dot{f}(0, a)^{\dagger}\left[f(0, a)^{\dagger}\right]^{-1}+\dot{f}(0, a)^{\dagger}\left[f(0, a)^{\dagger}\right]^{-1} S(0),
\end{gathered}
$$

with the $n \times n$ matrix $\mathcal{E}_{2}$ defined as

$$
\mathcal{E}_{2}:=2\left[\begin{array}{cc}
-\mathcal{A}_{2} \mathcal{A}_{1}^{-1} & 0 \\
\mathcal{E}_{3} & \left(\mathcal{D}_{1}-\mathcal{C}_{1} \mathcal{A}_{1}^{-1} \mathcal{B}_{1}\right) \mathcal{D}_{0}^{-1}
\end{array}\right],
$$

and with the $(n-\mu) \times \mu$ matrix $\mathcal{E}_{3}$ given by

$$
\mathcal{E}_{3}:=\left(\mathcal{C}_{1} \mathcal{A}_{1}^{-1} \mathcal{B}_{1} \mathcal{D}_{0}^{-1} \mathcal{C}_{1}-C_{1} \mathcal{A}_{1}^{-1} \mathcal{A}_{2}-\mathcal{D}_{1} \mathcal{D}_{0}^{-1} \mathcal{C}_{1}\right) \mathcal{A}_{1}^{-1}
$$

PROOF: In order to get (7.30), we use (7.14), (7.16), and (7.28) in (7.25). In a similar way, we obtain (7.33) by using (7.21) and (7.29) in (7.26). Finally, we obtain (7.35) by using (7.16), (7.21), (7.28), and (7.29) in (7.27).

Let us emphasize that in the expansions (7.30), (7.33), and (7.35) for $J(k), J(k)^{-1}$, and $S(k)$, respectively, we can construct explicitly the first two terms from knowledge of the matrix potential $V$ and the constant matrices $A$ and $B$ appearing in (1.3). For this purpose, we can first obtain $f(0, x)$ and $\dot{f}(0, x)$ by solving $(2.5)$ with the respective asymptotic conditions (2.14) and (2.18). We then get $J(0)$ from (5.2). Next, we can construct the constant matrices $P_{1}, P_{2}$, and $\mathcal{S}$ so that (7.17) is satisfied. Using (3.26), we can obtain the constant matrix $q_{1}(a)$, where $a$ is a nonnegative number for which the matrix $f(0, a)$ is invertible. Next, with the help of (2.38) and (2.39), we can get the constant matrices $\omega_{1}(0)$ and $\omega_{1}^{\prime}(0)$. We can construct $\varphi(0, a)$ by solving $(2.5)$ with the conditions in (2.45) by putting $k=0$ there. Then, via (7.11) and (7.12) we can get the constant 
matrices $\mathcal{R}$ and $F_{2}$. Next, with the help of (7.18)-(7.20), we obtain the constant matrices $\mathcal{A}_{1}, \mathcal{A}_{2}, \mathcal{B}_{1}, \mathcal{C}_{1}, \mathcal{D}_{0}$, and $\mathcal{D}_{1}$. We then have all the ingredients for the first two terms in each of the expansions (7.30), (7.33), and (7.35) because, as seen from (7.30)-(7.39), those coefficients can explicitly be obtained from the constant matrices $f(0, a), \dot{f}(0, a), P_{1}, P_{2}$, $\mathcal{S}, \mathcal{A}_{1}, \mathcal{A}_{2}, \mathcal{B}_{1}, \mathcal{C}_{1}, \mathcal{D}_{0}$, and $\mathcal{D}_{1}$

\section{AN EXPLICIT EXAMPLE}

In this section we illustrate our results on the small- $k$ asymptotics of the Jost matrix, the inverse of the Jost matrix, and the scattering matrix by presenting an explicit example. In the generic case we illustrate and verify the asymptotics in (5.1), (5.4), and (5.5). In the exceptional case we illustrate and verify the asymptotics given in (7.30), (7.33), and (7.35).

In our example we use the so-called Kirchhoff boundary conditions given by

$$
\psi_{1}(0)=\psi_{2}(0)=\psi_{3}(0), \quad \psi_{1}^{\prime}(0)+\psi_{2}^{\prime}(0)+\psi_{3}^{\prime}(0)=0,
$$

where $\psi_{j}(x)$ denotes the $j$ th component of the wavefunction $\psi(x)$ appearing in (1.3). Such boundary conditions are relevant in many application areas and may correspond, for example in quantum wires, to the continuity of the wavefunction and the conservation of the current at a junction.

Assume that the potential is given by

$$
V(x)=\left[\begin{array}{ccc}
\frac{32 e^{2 x}}{\left(4 e^{2 x}-1\right)^{2}} & 0 & 0 \\
0 & 0 & 0 \\
0 & 0 & 0
\end{array}\right]+\delta(x-1)\left[\begin{array}{lll}
0 & 0 & 0 \\
0 & 1 & 1 \\
0 & 1 & \gamma
\end{array}\right],
$$

where $\delta(x)$ denotes the Dirac delta distribution and $\gamma$ is a real parameter. We will see that the choice $\gamma=-31 / 77$ corresponds to $\operatorname{det}[J(0)]=0$ and any other $\gamma$ value corresponds to $\operatorname{det}[J(0)] \neq 0$. Suppose that the boundary parameter matrices $A$ and $B$ appearing in (1.3) 
are given by

$$
A=\left[\begin{array}{lll}
0 & 0 & 1 \\
0 & 0 & 1 \\
0 & 0 & 1
\end{array}\right], \quad B=\left[\begin{array}{ccc}
-1 & 0 & 0 \\
1 & -1 & 0 \\
0 & 1 & 0
\end{array}\right]
$$

so that we have the Kirchhoff boundary conditions given in (8.1). We can evaluate the Jost solution explicitly and obtain

$$
f(k, x)=\left\{\begin{array}{l}
{\left[\begin{array}{ccc}
f_{1} & 0 & 0 \\
0 & f_{2} & f_{3} \\
0 & f_{3} & f_{4}
\end{array}\right], \quad 0 \leq x \leq 1,} \\
{\left[\begin{array}{ccc}
f_{1} & 0 & 0 \\
0 & e^{i k x} & 0 \\
0 & 0 & e^{i k x}
\end{array}\right], \quad x \geq 1,}
\end{array}\right.
$$

where we have defined

$$
\begin{gathered}
f_{1}:=e^{i k x}\left(1+\frac{2 i}{(k+i)\left(4 e^{2 x}-1\right)}\right), \quad f_{2}:=e^{i k x}\left(1+\frac{i}{2 k}\right)-\frac{i}{2 k} e^{i k(2-x)}, \\
f_{3}:=\frac{i}{2 k} e^{i k x}-\frac{i}{2 k} e^{i k(2-x)}, \quad f_{4}:=e^{i k x}\left(1+\frac{i \gamma}{2 k}\right)-\frac{i \gamma}{2 k} e^{i k(2-x)} .
\end{gathered}
$$

Note that $f(k, 0)$ and $f^{\prime}(k, 0)$ are given by

$$
\begin{gathered}
f(k, 0)=\left[\begin{array}{ccc}
1+\frac{2 i}{3(k+i)} & 0 & 0 \\
0 & 1+\frac{i}{2 k}\left(1-e^{2 i k}\right) & \frac{i}{2 k}\left(1-e^{2 i k}\right) \\
0 & \frac{i}{2 k}\left(1-e^{2 i k}\right) & 1+\frac{i \gamma}{2 k}\left(1-e^{2 i k}\right)
\end{array}\right], \\
f^{\prime}(k, 0)=\left[\begin{array}{ccc}
i k-\frac{2(3 k+8 i)}{9(k+i)} & 0 & 0 \\
0 & i k-\frac{1}{2}\left(1+e^{2 i k}\right) & -e^{i k} \cos k \\
0 & -e^{i k} \cos k & i k-\frac{\gamma}{2}\left(1+e^{2 i k}\right)
\end{array}\right] .
\end{gathered}
$$


Using (8.3)-(8.5) in (4.2) we get the Jost matrix as

$$
J(k)=\left[\begin{array}{ccc}
-1-\frac{2 i}{3(k+i)} & 0 & -i k+\frac{2}{3}+\frac{10 i}{9(k+i)} \\
1+\frac{i}{2 k}\left(1-e^{2 i k}\right) & -1 & 1-i k+e^{2 i k} \\
\frac{1}{k} e^{i k} \sin k & 1+\frac{\gamma-1}{k} e^{i k} \sin k & -i k+\frac{\gamma+1}{2}\left(1+e^{2 i k}\right)
\end{array}\right] .
$$

The determinant of $J(k)$ has the small- $k$ asymptotics

$$
\operatorname{det}[J(k)]=\frac{31+77 \gamma}{9}+\frac{(128 \gamma-39) i k}{9}+\left(\frac{131}{27}-\frac{193 \gamma}{9}\right) k^{2}+O\left(k^{3}\right), \quad k \rightarrow 0 \text { in } \mathbf{C} .
$$

Thus, $\gamma=-31 / 77$ corresponds to the exceptional case, i.e. $\operatorname{det}[J(0)]=0$, and any other value of $\gamma$ yields a generic case, i.e. $\operatorname{det}[J(0)] \neq 0$. The small- $k$ limit of $J(k)$ is given by

$$
J(k)=J(0)+k \dot{J}(0)+O\left(k^{2}\right), \quad k \rightarrow 0 \text { in } \mathbf{C},
$$

with

$$
J(0)=\left[\begin{array}{ccc}
-\frac{5}{3} & 0 & \frac{16}{9} \\
2 & -1 & 2 \\
1 & \gamma & 1+\gamma
\end{array}\right], \quad \dot{J}(0)=\left[\begin{array}{ccc}
-\frac{2 i}{3} & 0 & \frac{i}{9} \\
i & 0 & i \\
i & i(\gamma-1) & i \gamma
\end{array}\right] .
$$

For the choice $\gamma=-31 / 77$ we get the asymptotics

$$
J(k)^{-1}=\frac{i}{k} M_{1}+M_{2}+O(k), \quad k \rightarrow 0 \text { in } \mathbf{C},
$$

where we have defined

$$
M_{1}:=\left[\begin{array}{ccc}
\frac{144}{6971} & -\frac{496}{6971} & \frac{1232}{6971} \\
\frac{558}{6971} & -\frac{1922}{6971} & \frac{4774}{6971} \\
\frac{135}{6971} & -\frac{465}{6971} & \frac{1155}{6971}
\end{array}\right], \quad M_{2}:=\left[\begin{array}{ccc}
-\frac{16095714}{48594841} & \frac{22880111}{145784523} & \frac{32930051}{145784523} \\
-\frac{10281837}{48594841} & -\frac{30462632}{145784523} & \frac{61380319}{145784523} \\
\frac{11927250}{48594841} & \frac{8244046}{48594841} & \frac{7573258}{48594841}
\end{array}\right],
$$

and similarly, for the scattering matrix we get

$$
S(k)=S(0)+k \dot{S}(0)+O\left(k^{2}\right), \quad k \rightarrow 0 \text { in } \mathbf{C},
$$


with

$$
\begin{gathered}
S(0)=\left[\begin{array}{rrr}
-\frac{6809}{6971} & -\frac{558}{6971} & \frac{1386}{6971} \\
-\frac{558}{6971} & -\frac{5049}{6971} & -\frac{4774}{6971} \\
\frac{1386}{6971} & -\frac{4774}{6971} & \frac{4887}{6971}
\end{array}\right], \\
\dot{S}(0)=\left[\begin{array}{ccc}
\frac{24111452 i}{48594841} & -\frac{8336928 i}{48594841} & -\frac{12952632 i}{48594841} \\
-\frac{8336928 i}{48594841} & \frac{95224498 i}{145784523} & \frac{111299650 i}{145784523} \\
-\frac{12952632 i}{48594841} & \frac{111299650 i}{145784523} & -\frac{124617494 i}{145784523}
\end{array}\right] .
\end{gathered}
$$

In the generic case, we have (8.9) with the relevant matrices specified as

$$
S(0)=-I_{3}, \quad \dot{S}(0)=\left[\begin{array}{ccc}
\frac{2 i(20 \gamma+7)}{77 \gamma+31} & -\frac{18 i \gamma}{77 \gamma+31} & -\frac{18 i}{77 \gamma+31} \\
-\frac{18 i \gamma}{77 \gamma+31} & \frac{62 i \gamma}{77 \gamma+31} & \frac{62 i}{77 \gamma+31} \\
-\frac{18 i}{77 \gamma+31} & \frac{62 i}{77 \gamma+31} & \frac{2 i(77 \gamma-46)}{77 \gamma+31}
\end{array}\right],
$$

where we recall that $I_{3}$ denotes the $3 \times 3$ identity matrix.

Let us now verify our results on the small- $k$ limits. In the generic case, i.e. when $\gamma \neq-31 / 77$, we will obtain the small- $k$ limits as described in Theorem 5.1. From (8.4) and (8.5) we get

$$
\begin{aligned}
& f(0,0)=\left[\begin{array}{ccc}
\frac{5}{3} & 0 & 0 \\
0 & 2 & 1 \\
0 & 1 & \gamma+1
\end{array}\right], \quad f^{\prime}(0,0)=\left[\begin{array}{ccc}
-\frac{16}{9} & 0 & 0 \\
0 & -1 & -1 \\
0 & -1 & -\gamma
\end{array}\right], \\
& \dot{f}(0,0)=\left[\begin{array}{ccc}
\frac{2 i}{3} & 0 & 0 \\
0 & i & i \\
0 & i & i \gamma
\end{array}\right], \quad \dot{f}^{\prime}(0,0)=\left[\begin{array}{ccc}
-\frac{i}{9} & 0 & 0 \\
0 & 0 & -i \\
0 & -i & i(1-\gamma)
\end{array}\right] \text {. }
\end{aligned}
$$

Using (8.2), (8.13), and (8.14) in (5.2) and (5.3) we obtain (8.6) with

$$
J(0)=\left[\begin{array}{ccc}
-\frac{5}{3} & 0 & \frac{16}{9} \\
2 & -1 & 2 \\
1 & \gamma & 1+\gamma
\end{array}\right], \quad \dot{J}(0)=\left[\begin{array}{ccc}
-\frac{2 i}{3} & 0 & \frac{i}{9} \\
i & 0 & i \\
i & i(\gamma-1) & i \gamma
\end{array}\right] \text {, }
$$


agreeing with the quantities given in (8.7). Similarly, using (8.15) in (5.5) we obtain (8.9) with $S(0)$ and $\dot{S}(0)$ agreeing with the quantities given in (8.10) and (8.11), respectively. Thus, we have verified the results of Theorem 5.1 in the generic case for our example.

Let us now verify, in the exceptional case i.e. when $\gamma=-31 / 77$, the small- $k$ asymptotics presented in Section 7. From (8.4) and (8.5) with $\gamma=-31 / 77$, we obtain

$$
\begin{gathered}
f(0,0)=\left[\begin{array}{ccc}
\frac{5}{3} & 0 & 0 \\
0 & 2 & 1 \\
0 & 1 & \frac{46}{77}
\end{array}\right], \quad f^{\prime}(0,0)=\left[\begin{array}{ccc}
-\frac{16}{9} & 0 & 0 \\
0 & -1 & -1 \\
0 & -1 & \frac{31}{77}
\end{array}\right], \\
\dot{f}(0,0)=\left[\begin{array}{ccc}
\frac{2 i}{3} & 0 & 0 \\
0 & i & i \\
0 & i & -\frac{31 i}{77}
\end{array}\right], \quad \dot{f}^{\prime}(0,0)=\left[\begin{array}{ccc}
-\frac{i}{9} & 0 & 0 \\
0 & 0 & -i \\
0 & -i & \frac{108 i}{77}
\end{array}\right] .
\end{gathered}
$$

Using (8.2), (8.4), and (8.5) in (5.2) and (5.3), we obtain (8.6) with

$$
J(0)=\left[\begin{array}{ccc}
-\frac{5}{3} & 0 & \frac{16}{9} \\
2 & -1 & 2 \\
1 & -\frac{31}{77} & \frac{46}{77}
\end{array}\right], \quad \dot{J}(0)=\left[\begin{array}{ccc}
-\frac{2 i}{3} & 0 & \frac{i}{9} \\
i & 0 & i \\
i & -\frac{108 i}{77} & -\frac{31 i}{77}
\end{array}\right]
$$

agreeing with the values given in (8.7) when $\gamma=-31 / 77$. The eigenvalues of $J(0)$ are obtained from (8.15) and are given by

$$
\lambda_{1}=0, \quad \lambda_{2}=\frac{-239+2 \sqrt{26273}}{231}, \quad \lambda_{3}=\frac{-239-2 \sqrt{26273}}{231},
$$

with respective eigenvectors

$$
v_{1}=\left[\begin{array}{c}
\frac{16}{15} \\
\frac{62}{15} \\
1
\end{array}\right], \quad v_{2}=\left[\begin{array}{c}
\frac{-73+\sqrt{26273}}{102} \\
\frac{2399+3 \sqrt{26273}}{1054} \\
1
\end{array}\right], \quad v_{3}=\left[\begin{array}{c}
\frac{-73-\sqrt{26273}}{102} \\
\frac{2399-3 \sqrt{26273}}{1054} \\
1
\end{array}\right] .
$$


We form the columns of the constant matrix $\mathcal{S}$ appearing in (7.17) by using (8.20) to have

$$
\mathcal{S}=\left[\begin{array}{ccc}
\frac{16}{15} & \frac{-73+\sqrt{26273}}{102} & \frac{-73-\sqrt{26273}}{102} \\
\frac{62}{15} & \frac{2399+3 \sqrt{26273}}{1054} & \frac{2399-3 \sqrt{26273}}{1054} \\
1 & 1 & 1
\end{array}\right]
$$

Using (8.18) and (8.21) in (7.17) we see that

$$
P_{1}=I_{3}, \quad P_{2}=I_{3}
$$

Note that the determinant of $f(0,0)$ given in $(8.16)$ is $25 / 77$ and hence not zero, and thus we can choose the constant $a$ appearing in (2.23) as zero. Then, using (8.18) and (8.21) in (2.38) and (2.39) we obtain

$$
\omega_{1}(0)=\left[\begin{array}{lll}
0 & 0 & 0 \\
0 & 0 & 0 \\
0 & 0 & 0
\end{array}\right], \quad \omega_{1}^{\prime}(0)=\left[\begin{array}{lll}
0 & 0 & 0 \\
0 & 0 & 0 \\
0 & 0 & 0
\end{array}\right] .
$$

Similarly, using (8.3) in (3.26) we obtain

$$
q_{1}(0)=\left[\begin{array}{ccc}
\frac{16}{15} & 0 & 0 \\
0 & -\frac{2}{5} & \frac{2438}{385} \\
0 & \frac{2438}{385} & -\frac{275162}{29645}
\end{array}\right]
$$

From (2.45) and (8.2) we obtain

$$
\varphi(0,0)=\left[\begin{array}{lll}
0 & 0 & 1 \\
0 & 0 & 1 \\
0 & 0 & 1
\end{array}\right]
$$

Then, using (8.2), (8.23)-(8.25) in (7.11) and (7.12) we obtain

$$
\mathcal{R}=\left[\begin{array}{ccc}
0 & 0 & \frac{3}{5} \\
0 & 0 & -\frac{31}{15} \\
0 & 0 & \frac{77}{15}
\end{array}\right], \quad F_{2}=\left[\begin{array}{ccc}
0 & 0 & -\frac{16}{25} \\
0 & 0 & -\frac{100}{3} \\
0 & 0 & \frac{70148}{1155}
\end{array}\right] .
$$


Next, using (8.18), (8.19), (8.21), and (8.22) in (7.17) and (7.18) we get

$$
\mathcal{D}_{0}=\left[\begin{array}{cc}
\frac{-239+2 \sqrt{26273}}{231} & 0 \\
0 & \frac{-239-2 \sqrt{26273}}{231}
\end{array}\right],
$$

and using (8.21), (8.22), and (8.26) in (7.19) and (7.20) we obtain

$$
\begin{aligned}
& \mathcal{A}_{1}=\left[\frac{6971 i}{623}\right], \quad \mathcal{B}_{1}=\left[\begin{array}{ll}
\frac{6971 i}{623} & \frac{6971 i}{623}
\end{array}\right], \quad \mathcal{C}_{1}=\left[\begin{array}{c}
-\frac{76268 i}{9345}-\frac{11541857 i}{9345 \sqrt{26273}} \\
-\frac{76268 i}{9345}+\frac{11541857 i}{9345 \sqrt{26273}}
\end{array}\right], \\
& \mathcal{D}_{1}=\left[\begin{array}{cc}
-\frac{76268 i}{9345}-\frac{11541857 i}{9345 \sqrt{26273}} & -\frac{76268 i}{9345}-\frac{11541857 i}{9345 \sqrt{26273}} \\
-\frac{76268 i}{9345}+\frac{11541857 i}{9345 \sqrt{26273}} & -\frac{76268 i}{9345}+\frac{11541857 i}{9345 \sqrt{26273}}
\end{array}\right] \\
& \mathcal{A}_{2}=\left[-\frac{427808}{3115}\right], \quad \mathcal{B}_{2}=\left[\begin{array}{ll}
-\frac{427808}{3115} & -\frac{427808}{3115}
\end{array}\right] \text {, } \\
& \mathcal{C}_{2}=\left[\begin{array}{c}
\frac{10180418}{102795}+\frac{7539081034}{513975 \sqrt{26273}} \\
\frac{10180418}{102795}-\frac{7539081034}{513975 \sqrt{26273}}
\end{array}\right] \\
& \mathcal{D}_{2}=\left[\begin{array}{ll}
\frac{10180418}{102795}+\frac{7539081034}{513975 \sqrt{26273}} & \frac{10180418}{102795}+\frac{7539081034}{513975 \sqrt{26273}} \\
\frac{10180418}{102795}-\frac{7539081034}{513975 \sqrt{26273}} & \frac{10180418}{102795}-\frac{7539081034}{513975 \sqrt{26273}}
\end{array}\right] .
\end{aligned}
$$

Thus, we have all the ingredients for the verification of the results presented in Theorem 7.6. Using (8.21), (8.22), (8.26)-(8.32) in (7.31), (7.32), (7.34), (7.36)-(7.39) we obtain

$$
\begin{gathered}
J(0)=\left[\begin{array}{ccc}
-\frac{5}{3} & 0 & \frac{16}{9} \\
2 & -1 & 2 \\
1 & -\frac{31}{77} & \frac{46}{77}
\end{array}\right], \quad \dot{J}(0)=\left[\begin{array}{ccc}
-\frac{2 i}{3} & 0 & \frac{i}{9} \\
i & 0 & i \\
i & -\frac{108 i}{77} & -\frac{31 i}{77}
\end{array}\right], \\
\mathcal{E}_{1}=\left[\begin{array}{ccc}
-\frac{16095714}{48594841} & \frac{22880111}{145784523} & \frac{32930051}{145784523} \\
-\frac{10281837}{48594841} & -\frac{30462632}{145784523} & \frac{61380319}{145784523} \\
\frac{11927250}{48594841} & \frac{8244046}{48594841} & \frac{7573258}{48594841}
\end{array}\right]
\end{gathered}
$$




$$
\begin{aligned}
\mathcal{E}_{2}= & {\left[\begin{array}{ccc}
\frac{-\frac{855616 i}{34855}}{85516 i(2003789164+11541857 \sqrt{26273})} & 0 & 0 \\
\frac{855754919319475}{25616 i(2003789164-11541857 \sqrt{26273})} & 0 & 0
\end{array}\right], } \\
\mathcal{E}_{3}= & {\left[\begin{array}{ccc}
\frac{427808 i(2003789164+11541857 \sqrt{26273})}{95754919319475} \\
\frac{427808 i(2003789164-11541857 \sqrt{26273)}}{95754919319475}
\end{array}\right], } \\
& {\left[\begin{array}{ccc}
-\frac{6809}{6971} & -\frac{558}{6971} & \frac{1386}{6971} \\
-\frac{558}{6971} & -\frac{5049}{6971} & -\frac{4774}{6971} \\
\frac{1386}{6971} & -\frac{4774}{6971} & \frac{4887}{6971}
\end{array}\right], } \\
\dot{S}(0)= & {\left[\begin{array}{ccc}
\frac{24111452 i}{48594841} & -\frac{8336928 i}{48594841} & -\frac{12952632 i}{48594841} \\
-\frac{8336928 i}{48594841} & \frac{95224498 i}{145784523} & \frac{111299650 i}{145784523} \\
-\frac{12952632 i}{48594841} & \frac{111299650 i}{145784523} & -\frac{124617494 i}{145784523}
\end{array}\right] . }
\end{aligned}
$$

Finally, by using (8.33)-(8.37), we evaluate (7.30), (7.33), and (7.35) and obtain the small$k$ expansions for $J(k), J(k)^{-1}$, and $S(k)$, respectively, which all agree with the expansions given in (8.6), (8.8), and (8.9).

Acknowledgments. The research leading to this article was supported in part by Consejo Nacional de Ciencia y Tecnología (CONACYT) under project CB2008-99100-F and by the Department of Defense under grant number DOD-BC063989.

\section{References}

[1] Z. S. Agranovich and V. A. Marchenko, The inverse problem of scattering theory, Gordon and Breach, New York, 1963. 
[2] T. Aktosun, Factorization and small-energy asymptotics for the radial Schrödinger equation, J. Math. Phys. 41, 4262-4270 (2000).

[3] T. Aktosun and M. Klaus, Small-energy asymptotics for the Schrödinger equation on the line, Inverse Problems 17, 619-632 (2001).

[4] T. Aktosun, M. Klaus, and C. van der Mee, Small-energy asymptotics of the scattering matrix for the matrix Schrödinger equation on the line, J. Math. Phys. 42, 4627-4652 (2001).

[5] T. Aktosun, M. Klaus, and R. Weder, Small-energy analysis for the self-adjoint matrix Schrödinger operator on the half line, J. Math. Phys. 52, 102101 (2011).

[6] T. Aktosun and R. Weder, High-energy analysis and Levinson's theorem for the selfadjoint matrix Schrödinger operator on the half line, J. Math. Phys. 54, 012108 (2013).

[7] G. Berkolaiko, R. Carlson, S. A. Fulling, and P. Kuchment (eds.), Quantum graphs and their applications, Contemporary Mathematics, 415, Amer. Math. Soc., Providence, RI, 2006.

[8] J. Boman and P. Kurasov, Symmetries of quantum graphs and the inverse scattering problem, Adv. Appl. Math. 35, 58-70 (2005).

[9] E. A. Coddington and N. Levinson, Theory of differential equations, New York, McGraw-Hill, 1955.

[10] P. Deift and E. Trubowitz, Inverse scattering on the line, Commun. Pure Appl. Math. 32, 121-251 (1979).

[11] P. Exner, J. P. Keating, P. Kuchment, T. Sunada, and A. Teplyaev (eds.), Analysis on graphs and its applications, Proc. Symposia in Pure Mathematics, 77, Amer. Math. Soc., Providence, RI, 2008.

[12] L. D. Faddeev, Properties of the S-matrix of the one-dimensional Schrödinger equa- 
tion, Amer. Math. Soc. Transl. 65 (ser. 2), 139-166 (1967).

[13] N. I. Gerasimenko, The inverse scattering problem on a noncompact graph, Theoret. Math. Phys. 75, 460-470 (1988).

[14] N. I. Gerasimenko and B. S. Pavlov, A scattering problem on noncompact graphs, Theoret. Math. Phys. 74, 230-240 (1988).

[15] B. Gutkin and U. Smilansky, Can one hear the shape of a graph? J. Phys. A 34, 6061-6068 (2001).

[16] M. S. Harmer, Inverse scattering for the matrix Schrödinger operator and Schrödinger operator on graphs with general self-adjoint boundary conditions, ANZIAM J. 44, 161168 (2002).

[17] M. S. Harmer, The matrix Schrödinger operator and Schrödinger operator on graphs, Ph.D. thesis, University of Auckland, New Zealand, 2004.

[18] M. Harmer, Inverse scattering on matrices with boundary conditions, J. Phys. A 38, 4875-4885 (2005).

[19] M. Klaus, Low-energy behaviour of the scattering matrix for the Schrödinger equation on the line, Inverse Problems 4, 505-512 (1988).

[20] M. Klaus, Exact behavior of Jost functions at low energy, J. Math. Phys. 29, 148-154 (1988).

[21] V. Kostrykin and R. Schrader, Kirchhoff's rule for quantum wires, J. Phys. A 32, 595-630 (1999).

[22] V. Kostrykin and R. Schrader, Kirchhoff's rule for quantum wires. II: The inverse problem with possible applications to quantum computers, Fortschr. Phys. 48, 703-716 (2000).

[23] P. Kuchment, Quantum graphs. I. Some basic structures, Waves Random Media 14, 
S107-S128 (2004).

[24] P. Kuchment, Quantum graphs. II. Some spectral properties of quantum and combinatorial graphs, J. Phys. A 38, 4887-4900 (2005).

[25] P. Kurasov and M. Nowaczyk, Inverse spectral problem for quantum graphs, J. Phys. A 38, 4901-4915 (2005).

[26] P. Kurasov and F. Stenberg, On the inverse scattering problem on branching graphs, J. Phys. A 35, 101-121 (2002). 